ARTICLE

https://doi.org/10.1038/s41467-019-11043-w

\title{
Sensory cortex wiring requires preselection of short- and long-range projection neurons through an Egr-Foxg1-COUP-TFI network
}

\author{
Pei-Shan Hou ${ }^{1,2,3}$, Goichi Miyoshi (i] ${ }^{4}$ \& Carina Hanashima ${ }^{1,2,3}$
}

The bimodal requisite for a genetic program and external stimuli is a key feature of sensory circuit formation. However, the contribution of cell-intrinsic codes to directing sensoryspecific circuits remains unknown. Here, we identify the earliest molecular program that preselects projection neuron types in the sensory neocortex. Mechanistically, Foxg1 binds to an H3K4me1-enriched enhancer site to repress COUP-TFI, where ectopic acquisition of Foxg1 in layer 4 cells transforms local projection neurons to callosal projection neurons with pyramidal morphologies. Removal of Foxg1 in long-range projection neurons, in turn, derepresses COUP-TFI and activates a layer 4 neuron-specific program. The earliest segregation of projection subtypes is achieved through repression of Foxg1 in layer 4 precursors by early growth response genes, the major targets of the transforming growth factor- $\beta$ signaling pathway. These findings describe the earliest cortex-intrinsic program that restricts neuronal connectivity in sensory circuits, a fundamental step towards the acquisition of mammalian perceptual behavior.

\footnotetext{
${ }^{1}$ Department of Biology, Faculty of Education and Integrated Arts and Sciences, Waseda University, Shinjuku-ku, Tokyo 162-8480, Japan. ${ }^{2}$ Graduate Schoo of Advanced Science and Engineering, Waseda University, Shinjuku-ku, Tokyo 162-8480, Japan. ${ }^{3}$ Laboratory for Neocortical Development, RIKEN Center for Developmental Biology, Kobe 650-0047, Japan. ${ }^{4}$ Department of Neurophysiology, Tokyo Women's Medical University School of Medicine, Shinjuku-ku, Tokyo 162-8666, Japan. Correspondence and requests for materials should be addressed to C.H. (email: hanashima@waseda.jp)
} 
S ince the discovery of Brodmann areas ${ }^{1}$, protomap ${ }^{2}$ and protocortex ${ }^{3}$ hypotheses have argued the contribution of intrinsic vs. extrinsic regulation in establishing sensoryspecific circuits in the cerebral cortex. In these models, the protomap model supports the cortex-intrinsic origin of area specification, whereas the protocortex model argues that afferent projections relayed through the thalamus lie central to the establishment of sensory areas. At the cellular level, sensory inputs received by layer 4 (L4) granular neurons are delivered locally to layer $2 / 3$ (L2/3) intracortical projection neurons, which finally propagate to subcortical targets through long-range axons of layer 5 (L5) subcerebral projection neurons (SCPNs) ${ }^{4,5}$. Thus, L4 neurons serve as a central gateway for establishing sensory circuits to initiate higher order information processing.

While these two models propose complementary roles in sensory circuit formation ${ }^{2,6,7}$, recent work has emphasized the importance of extrinsic regulation, in which manipulation of inputs through thalamic innervation ${ }^{8,9}$, neuronal activity ${ }^{10}$, and cell positioning ${ }^{11}$ perturb the number of L4 neurons generated. Theoretically, however, recruitment of thalamic axons to their precise targets must involve a preselection mechanism of cortical layer neurons to guide these axon terminals, implying that a cortex-intrinsic mechanism underlies L4 development. Consistent with this view, blockage of sensory innervation ${ }^{6}$, neuronal transmitter depletion ${ }^{12}$, and rerouting of thalamic axons ${ }^{13}$ all fail to eliminate Ror $\beta$-expressing neurons, a hallmark of L4 thalamic input neurons. Furthermore, alteration in cell distribution results in correct L4 neuron specification and sensory circuit activation ${ }^{9}$, implying that the identity of L4 neurons is established independent of their extracellular environment. However, to date, the intrinsic machinery that preselects L4 neurons from long-range projection neurons to restrict their competence to attract thalamic innervation has remained unknown.

In contrast to the poor understanding of the ontogeny of L4 neurons, studies over the last decade have uncovered a core gene regulatory network that delineates long-range projection neuron subtypes of the neocortex ${ }^{14}$. In particular, corticofugal projection neurons (CFuPNs) and callosal projection neurons (CPNs) are produced through sequential derepression of key transcription factors (TFs) expressed in the developing neocortex ${ }^{14-19}$. In this scheme, the onset of Foxg1 expression in progenitor cells suppresses a default transcriptional network to initiate a SCPNspecific program ${ }^{20,21}$. Subsequent transition from SCPN to CPN production is achieved by activation of Satb2 expression, which is mediated through SCPN-derived feedback signals ${ }^{17,20,21}$. Consequently, these gene networks have revealed transcriptional cascades that direct the sequential production of layer $1,6,5$, and $2 / 3$ neurons in the neocortex; however, L4 sensory input neurons have been excluded from this network. Indeed, in contrast to long-range $\mathrm{CFuPNs}{ }^{22}$ and $\mathrm{CPNs}^{17}$, TFs unique to thalamic input L4 local projection neurons are underrepresented, with the exception of the orphan nuclear receptor Ror $\beta$, the only common marker identified for L4 neurons. Ror $\beta$ manipulation affects Brn1/2 expression ${ }^{23}$ and thalamic innervation ${ }^{24}$; however, the onset of Ror $\beta$ expression in L4 neurons appears relatively late during corticogenesis ${ }^{25}$. Moreover, inhibition of thalamocortical innervation does not eliminate Ror $\beta$ expression in L4 neurons ${ }^{6,8}$, indicating that the induction of Ror $\beta$ requires a cortex-intrinsic regulatory mechanism.

In this study, we found that the coordination between two highly evolutionarily conserved TFs susceptible to neurological disorders, the forkhead-box family gene Foxg1 and the nuclear hormone receptor COUP-TFI/Nr2f1, reciprocally regulates the earliest specification of long- and short-range projection neurons in the neocortex. Ectopic expression of Foxg1 or loss of COUPTFI expression attenuates the acquisition of L4 fate and induces a long-range projection neuron-specific program. COUP-TFI gainof-function (GOF), in turn, alters distal projection neurons to acquire a L4 neuron identity. We further demonstrate that downregulation of Foxg1 is the key event for L4 neuron specification mediated through COUP-TFI. This early segregation of projection subtypes is achieved through repression of Foxg1 by early growth response (Egr) genes, the major target of the transforming growth factor- $\beta$ (TGF $\beta$ ) signaling pathway activated in L4 precursors. These results identify the earliest intrinsic program that confers unique laminar and hodological properties in the neocortex, a fundamental step for the formation of sensoryspecific circuits.

\section{Results}

Reciprocal COUP-TFI and Foxg1 expression in laminar subtypes. To reveal the gene regulatory network that delineates thalamic input local projection neurons from long-range projection neurons, we performed an in silico screen for TFs expressed in a complementary manner between these laminar subtypes. The transcriptomic profile across cortical layers in the mouse cortex ${ }^{26}$ (http://genserv.anat.ox.ac.uk/layers) revealed enriched Foxg1 expression in L2/3 CPNs and L5 long-range SCPNs but low expression in thalamic input (L4) neurons (Fig. 1a), indicating its differential roles in the development of long (layers $2 / 3 / 5$ ) vs. short-range (L4) projection neurons. To assess the repression targets of Foxg1 that distinguish between these projection types, we utilized transcriptome data that manipulated Foxg1 expression in vivo during corticogenesis ${ }^{20}$ (Fig. 1b-d). Among the significantly downregulated genes upon Foxg1 induction (Fig. 1c, d), COUP-TFI/Nr2f1, an evolutionarily conserved orphan nuclear receptor gene, was found among the cluster of genes that exhibited the fastest and significant downregulation in gene expression (Fig. 1d). We next examined the expression of Foxg1 and COUP-TFI at the mid-corticogenesis period, which demonstrated mutual expression at E15.5 (Fig. 1e-e $\mathrm{e}^{\prime \prime}$ ). Temporal dynamics of Foxg1 and COUP-TFI expression showed that at E11.5, Foxg1 was mainly detected in progenitor cells of the ventricular zone (VZ), whereas COUP-TFI was expressed in a subpopulation of preplate cells (Fig. $\left.1 \mathrm{f}-\mathrm{f}^{\prime \prime}\right)^{27}$. Notably, at the cellular level, cells with high COUP-TFI expression exhibited low or no Foxg1 expression (Fig. 1f- $\mathrm{f}^{\prime \prime}$ ). At E13.5, COUP-TFI was scattered in the VZ and weakly expressed in some progenitor cells, whereas Foxg1 was broadly expressed in the progenitor cells. In the cortical plate $(\mathrm{CP})$, cells in the most superficial region of the CP expressed high levels of COUP-TFI, whereas other cells that expressed Foxg1 showed low or no COUP-TFI expression (Fig. 1g-g"). Immunohistochemistry detected Foxg1-negative COUP-TFI-positive cells in the marginal zone as in earlier stages, whereas Foxg1 and COUP-TFI were coexpressed in the deeper portion of the CP (Fig. 1h-h"). In contrast, double immunohistochemistry/in situ hybridization revealed that Foxg1 mRNA is absent in subplate and layer 6 corticothalamic projection neurons (CThPNs) (Fig. 1e-e $\mathrm{e}^{\prime \prime}$ ), indicating the perdurance of Foxg1 protein but lack of transcription activation in this population. Notably, many COUP-TFI-positive Foxg1-negative cells were detected in the intermediate zone at this stage (Fig. 1e-e", $\left.\mathrm{g}-\mathrm{h}^{\prime \prime}\right)$. On postnatal day $(\mathrm{P}) 1$, when neurogenesis has completed but L2/3 cortical neurons are still migrating, Foxg1 was widely expressed in CP neurons at variable levels but absent in Cajal-Retzius cells in the marginal zone and SP neurons (Fig. 1i-i"). At $\mathrm{P} 4$, when all projection neurons have arrived in the $\mathrm{CP}, \mathrm{L} 2 / 3$ cells expressed high Foxg1 and low COUP-TFI. Notably, Foxg1 and COUP-TFI showed complementary expression in L5 neurons, in which the lower part of L5 cells (L5b) expressed high Foxg1 with low or no COUP-TFI expression, and the upper part of L5 cells 
a

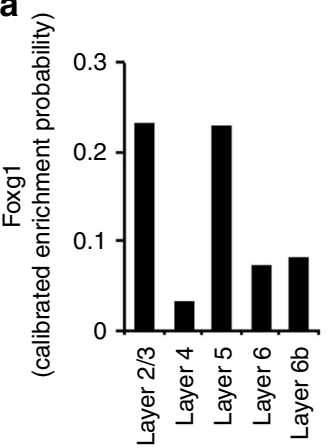

b

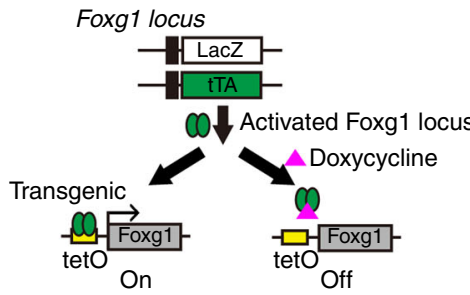

C

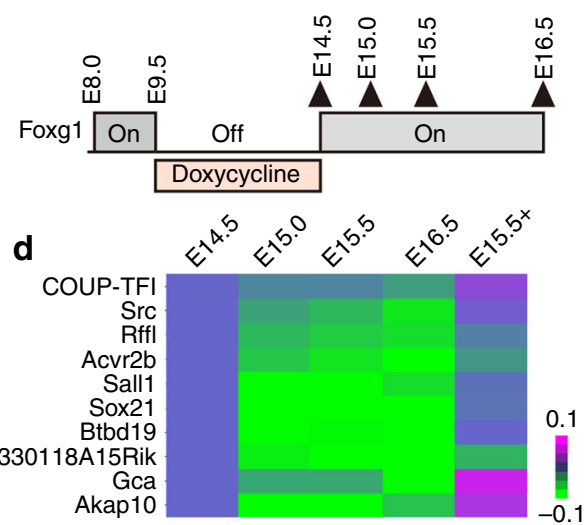

E15.5 COUP-TFI Foxg1 mRNA Nuclei
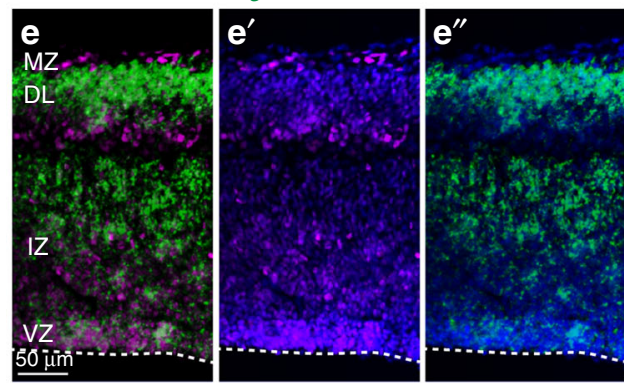

COUP-TFI Foxg1 Nuclei

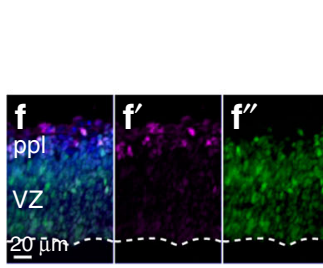

E11.5

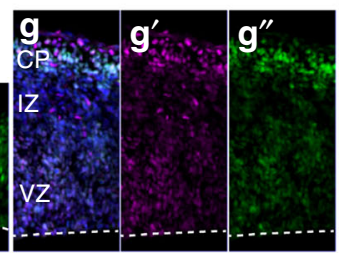

E13.5

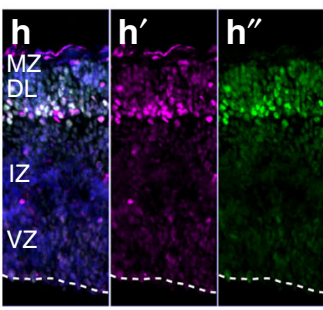

E15.5

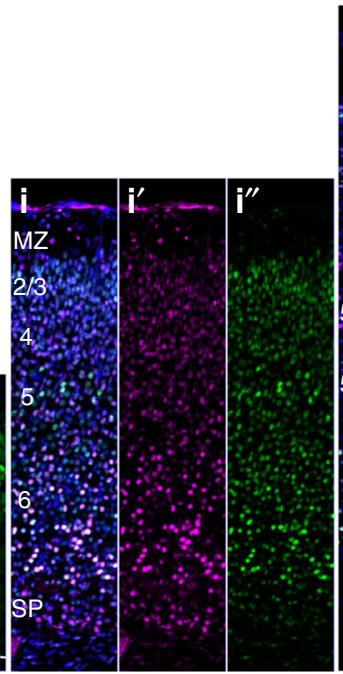

P1

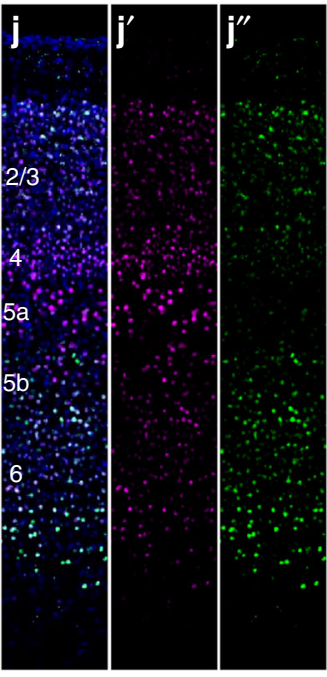

P4

Fig. 1 Reciprocal expression of COUP-TFI and Foxg1 in the developing neocortex. a Calibrated enrichment probability for Foxg1 expression across cortical layers in the adult mouse somatosensory cortex (http://genserv.anat.ox.ac.uk). b Schematic diagram showing the strategy of Foxg1 expression manipulation by doxycycline administration. In the absence of doxycycline, tet-transactivator (tTA) protein binds to tetO promoter to activate Foxg1 transgene expression. In the presence of doxycycline, doxycycline binds to tTA protein to prevent the activation of transgenic Foxg1 expression. c Schematic diagram showing the timing of doxycycline administration and the corresponding Foxg1 expression. Samples were collected at indicated time points shown in closed arrowheads. d Hierarchical clustering using the complete linkage method with Euclidean distance. Heatmap represents the gene cluster that exhibited rapid expression downregulation upon Foxg1 induction by doxycycline administration. e-e" Complementary expression of Foxg1 mRNA (green) by in situ hybridization and COUP-TFI protein (red) immunohistochemistry in E15.5 mouse cortex. Dashed lines indicate the ventricular surface. $\mathbf{f}-\mathbf{j}^{\prime \prime}$ Developmental expression of COUP-TFI (red) and Foxg1 (green) in E11.5 (f-f'f), E13.5 ( $\left.\mathbf{g}-\mathbf{g}^{\prime \prime}\right)$, E15.5 (h-h"), P1 (i-i-i"), and P4 (j-j") wild-type cortices. Mouse anti-COUP-TFI (Perseus) and Rabbit anti-Foxg1 (TaKaRa) antibodies were used. Embryonic tissues were processed at the identical condition using cryosections and postnatal tissues were perfused prior to fixation using floating sections. Dashed lines indicate the ventricular surface. VZ ventricular zone, IZ intermediate zone, CP cortical plate, MZ marginal zone, DL deep layer

(L5a) expressed low or no Foxg1 but high COUP-TFI expression. L4 cells were significantly enriched in COUP-TFI expression, whereas only the upper-most L4 cells expressed Foxg1 (Fig. 1j-j"). Thus, Foxg1 and COUP-TFI exhibit dynamic and complementary expression in cortical precursors and postmitotic neurons, indicating their reciprocal function in cortical laminar subtypes.

We next examined whether this mutual expression of Foxg1 and COUP-TFI is achieved through cell-autonomous or non-cell autonomous mechanisms. We introduced Foxg1 into the developing neocortex and analyzed these neurons 1 day after in utero electroporation (IUE) (Fig. 2a). Immunostaining revealed significant decrease in COUP-TFI expression in Foxg1overexpressed cells (Fig. 2b, c). In contrast, conditional removal of Foxg1 using Emx1 $1^{\mathrm{Cre} /+}$ mice $^{28}$ showed increased COUP-TFI expression in EGFP-positive cells (Foxg1 cKO in Fig. 2d, e), suggesting that COUP-TFI is negatively regulated by Foxg1 in neocortical precursor cells.

We next examined the regulatory machinery responsible for this rapid response of COUP-TFI to Foxg1 expression and their mutual expression in cortical precursors. Based on previous Foxg1-ChIP sequencing analysis using embryonic mouse neocortex (Fig. 2f ${ }^{20}$, we identified two putative Foxg1 binding sites (PBS1 and PBS2) within and near the COUP-TFI gene (Fig. 2f). We then performed Foxg1-qChIP analysis using E14.5 cortices and demonstrated specific binding of Foxg1 to one of the putative binding sites, PBS1, using Foxg1 knockout neocortical cells for a comparison (Fig. 2g). Notably, this region was enriched with H3K4me1, indicating an enhancer-like property (Fig. 2g). To 
a

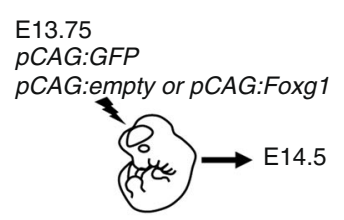

d

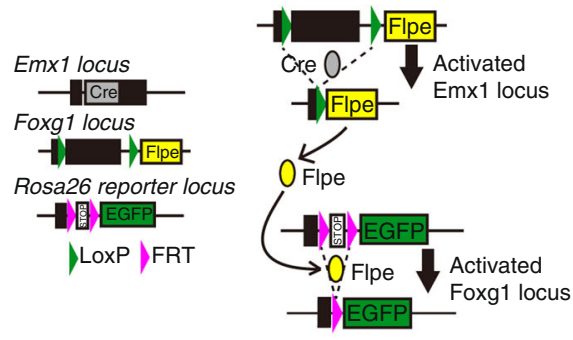

b

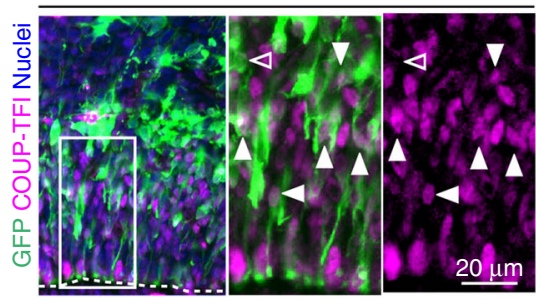

Foxg1 GOF

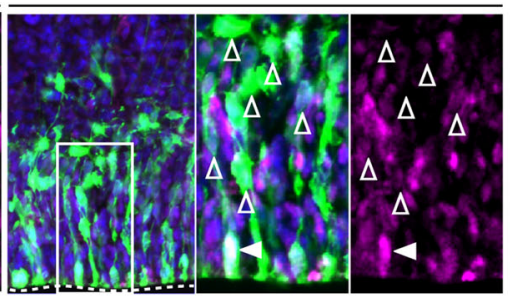

C

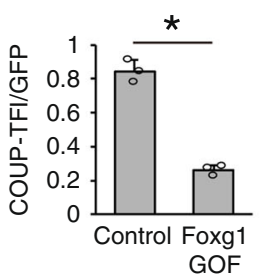

f

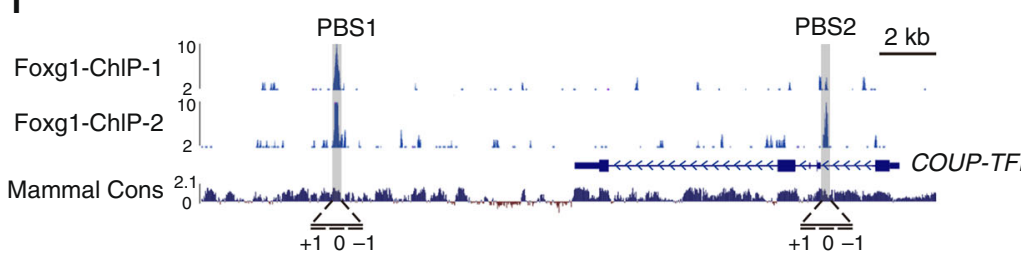

g

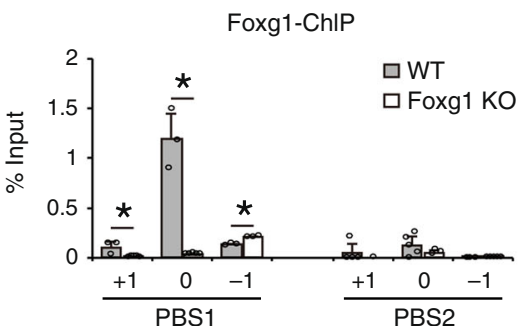

e $\quad E 14.5$

Control $\left(E m \times 1^{\text {Cre }} ;\right.$ Foxg $\left.{ }^{f / /+}\right)$

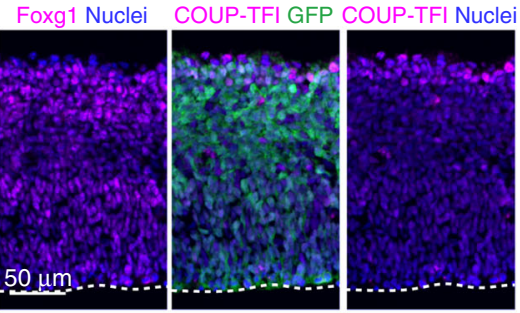

Foxg1 cKO $\left(E m \times 1^{C r e} ;\right.$ Foxg $\left.1^{\text {fl/fl }}\right)$

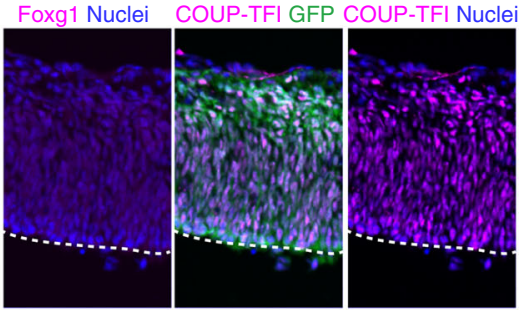

h RLU (relative to COUP-TFIpro)

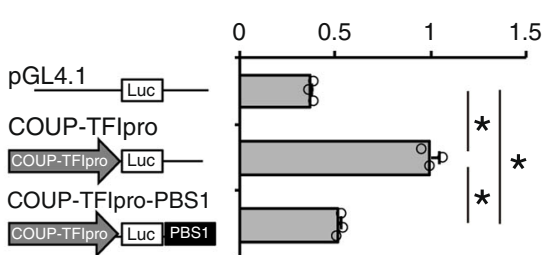

i

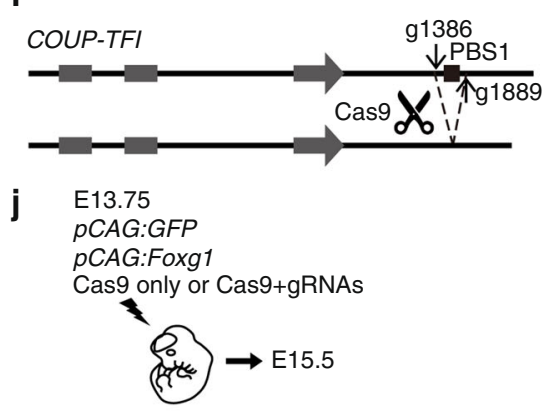

H3K4me1-ChIP

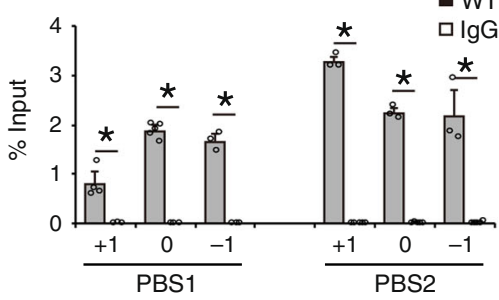

$\mathbf{k}$

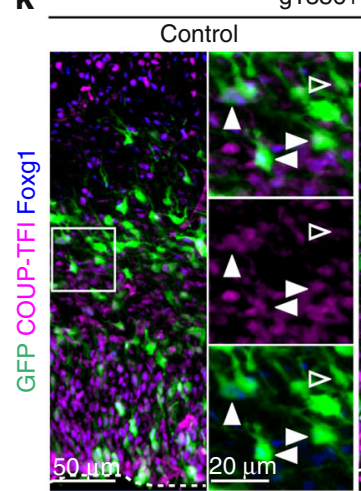

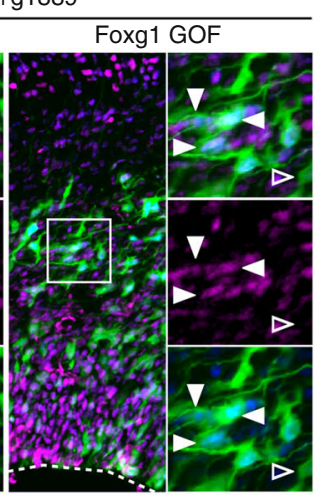

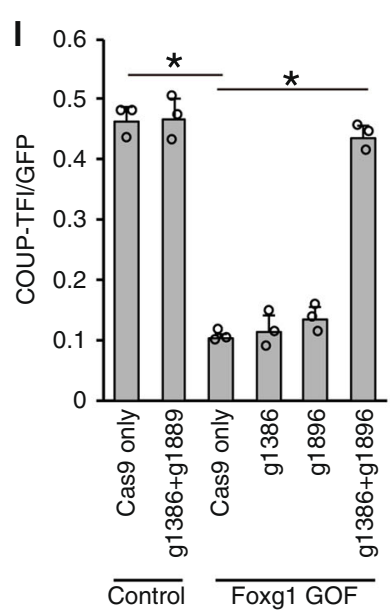

assess whether this enhancer-like region, PBS1, is targeted by Foxg1 to regulate the activity of the COUP-TFI promoter, we performed an in vitro reporter assay (Fig. $2 \mathrm{~h}$ ) that indicated that the presence of the Foxg1-targeted enhancer-like region (PBS1) suppresses the activity of the COUP-TFI promoter (Fig. 2h). To further confirm Foxg1-mediated COUP-TFI regulation in vivo, we introduced two gRNAs (g1386 and g1889) with Cas9 to remove the 503-bp PBS1 region in the E13.75 cortex using IUE and analyzed the embryos at E15.5 (Fig. 2i-l). In contrast to the loss of COUP-TFI expression in conditions of Foxg1 GOF alone (Fig. 2a-c), COUP-TFI expression was maintained under Foxg1 overexpression conditions when the Foxg1-targeted region was removed by the two sgRNAs compared with either of the sgRNAs introduced alone (Fig. 2k, 1 and Supplementary Fig. 1). Taken together, these results demonstrate that Foxg1 suppresses COUPTFI expression in the developing neocortex through direct binding to the PBS1 enhancer.

COUP-TFI directs layer 4 fate. We next investigated the consequence of this mutual expression in the specification of neocortical laminar subtypes. Based on our examination, L4 cells 
Fig. 2 Foxg1 suppresses COUP-TFI through an H3K4me1-enriched enhancer. a Schematic diagram of in utero electroporation. b COUP-TFI (red) and GFP (green) immunohistochemistry of E14.5 cortices. Closed arrowheads indicate GFP cells with COUP-TFI expression, open arrowheads indicate GFP cells without COUP-TFI expression. Dashed lines indicate the ventricular surface. c Quantitative analysis of the percentage ( \pm SEM) of GFP cells that express COUP-TFI. Brains were introduced with pCAG:GFP and pCAG:empty (Control) or pCAG:GFP and pCAG:Foxg1 (Foxg1 GOF). d Schematic diagram showing the strategy of conditional Foxg1 loss-of-function (Foxg1 cKO) using Emx1 Cre/+ mice. e Foxg1 (red), GFP (green), COUP-TFI (red), and Hoechst 33342 (blue) staining of E14.5 cortices. Dashed lines indicate the ventricular surface. f Schematic view of the COUP-TFI gene loci (chr13:78,316,360-78,342,718, UCSC genome browser, NCBI37/mm9). The data represent two independent ChIP-seq analyses (Foxg1-ChIP-1 and Foxg1-ChIP-2 from Kumamoto et al.20). Mammal Cons represents Placental Mammal Basewise Conservation by PhyloP. MACS peaks overlapping in the two ChIP-seq analyses are marked in gray boxes. Two putative binding sites, PBS1 and PBS2, and primers designed for ChIP-qPCR analysis are indicated (see Methods for details). $\mathbf{g}$ ChIP-qPCR analyses of PBS1 and PBS2 of cells isolated from E14.5 neocortex using anti-Foxg1 (TaKaRa) and anti-H3K4me1 (Abcam) antibodies. For negative controls, FoxglLacZ/LacZ (Foxg1 KO) neocortex (Foxg1-ChIP analysis) and IgG (H3K4me1-ChIP analysis) were used. h Quantitative COUP-TFI promoter activity $( \pm$ SEM) in the presence of PBS1 by in vitro reporter analysis in U87 human glioblastoma cell line. Value indicates RLU compared to COUPTFlpro (COUPTFI promoter) only. i Schematic diagram showing two gRNAs, g1386 and g1889, designed to target PBS1. j Schematic diagram of in utero electroporation. k Foxg1 (blue), GFP (green), and COUP-TFI (red) staining of E15.5 cortices. Closed arrowheads indicate GFP cells with COUP-TFI expression and open arrowheads indicate GFP cells without COUP-TFI expression. Brains were introduced with $p$ CAG:GFP and $p$ CAG:empty (Control) or $p$ CAG:GFP and $p$ CAG: Foxg1 (Foxg1 GOF). Dashed lines indicate the ventricular surface. I Quantitative analysis of the percentage ( \pm SEM) of GFP cells that express COUP-TFI. * indicates $P$ value $<0.05$ by Student's $t$-test. Source data are provided as a Source Data file

express low Foxg1 but high COUP-TFI (Fig. $\left.1 \mathrm{j}-\mathrm{j}^{\prime \prime}\right)$, and Foxg1suppresses COUP-TFI expression (Fig. 2). Therefore, we hypothesized that COUP-TFI expression is necessary for cells to acquire L4 fate. Because COUP-TFI is known to regulate cortical regionalization ${ }^{29-32}$, we attempted to manipulate COUP-TFI expression without attenuating cortical area establishment. To this end, we introduced the CRISPR/Cas9 system to disrupt the COUP-TFI gene together with the GFP reporter to label future L4 cells using IUE (Fig. 3a). Two pairs of gRNAs targeted to the first or second exon of the COUP-TFI gene were cloned into constructs containing the Cas 9 gene (Fig. 3a). These constructs were introduced into the E13.75 mouse neocortex by IUE to target future L4 cells (Fig. 3b). Two days after the introduction, COUPTFI expression in GFP-expressing cells was downregulated in the presence of gRNAs (Fig. 3c). Quantification of COUP-TFIexpressing cells among GFP-positive cells showed $76.8 \%$ and $71.2 \%$ reduction at E15.5 (Fig. 3d) and $87.9 \%$ and $92.8 \%$ reduction at P7 (Supplementary Fig. 2) with the introduction of g14 + g216 and g864 + g890, respectively, indicating efficient disruption of the COUP-TFI gene using the CRISPR/Cas9 system.

We next analyzed the cell-autonomous requirement of COUPTFI on L4 cell fate in the primary somatosensory cortex at P7, the timing of which positioning and thalamocortical connectivity of L4 cells has been established (Fig. $3 \mathrm{e}-\mathrm{u}$ ). In the control cortex, the majority of GFP-positive cells were located in somatosensory barrels with high Ror $\beta$ expression and showed spiny stellate morphology (Fig. 3e-e $\mathrm{e}^{\prime \prime}, \mathrm{h}, \mathrm{m}, \mathrm{n}, \mathrm{q}$ ). However, COUP-TFIdeficient cells were excluded from the barrels and extended a thick apical dendrite, resembling a pyramidal-like morphology (Fig. $3 \mathrm{f}-\mathrm{h}, \mathrm{m}$ ). These GFP-positive cells extended long-distance callosal axons through the corpus callosum and projected to the contralateral side (Fig. $3 \mathrm{j}-1$ ). The majority of COUP-TFI knockout cells lost Ror $\beta$ expression and in turn gained Brn2 and Satb2 expression (Fig. $3 \mathrm{n}-\mathrm{u}$ ). A proportion of COUP-TFI knockout cells lost upper-layer neuron identity as indicated by loss of Cux1 expression; however, there was no increase in the expression of Ctip2, a marker of deep-layer long-range SCPNs (Fig. 3u). Together, the morphological and molecular identity indicated the primary and cell-autonomous role of COUP-TFI in controlling L4 fate, which secondarily affects sensory area specification ${ }^{33}$.

Foxg1 attenuates layer 4 fate. Loss of COUP-TFI impairs L4 fate acquisition (Fig. 3), and Foxg1 directly binds to the COUP-TFI gene to suppress its expression (Fig. 2f-l); thus, the low Foxg1 expression in L4 cells (Fig. $1 \mathrm{j}-\mathrm{j}^{\prime \prime}$ ) implies that Foxg1 may negatively regulate the fate of L4 neurons through COUP-TFI repression. We thus introduced the Foxg1 gene with the CAG promoter together with the GFP reporter into E13.75 progenitor cells (Fig. 4a). Examination at P7 showed control cells positioned in somatosensory barrels with spiny stellate morphology (Fig. 4b, $\left.\mathrm{b}^{\prime}, \mathrm{d}, \mathrm{d}^{\prime}, \mathrm{e}\right)$. In contrast, cells that overexpressed Foxg1 as indicated by GFP-positive cells showed pyramidal cell morphology with apical dendrites (Fig. 4c, c', d, d') and changes in cell positioning (Fig. 4e). These neurons extended long-range projections through the corpus callosum (Supplementary Fig. 5c, $c^{\prime}$, d), which was rarely observed in control GFP neurons (Supplementary Fig. 5b, b', d). Quantitative analysis showed that $C A G$ : Foxg1-overexpressing cells lost Ror $\beta$ expression and gained Brn2 and Satb2 expression (Fig. 4g, g', j, j', m, m', o), as opposed to control cells that exhibited a Ror $\beta^{\text {high, }}$ Brn2 $2^{\text {low }}$, and Satb2 $2^{\text {low }}$ pattern (Fig. 4f, f', i, i', l, l', o). In addition, conditional Foxg1 overexpression using the NeuroD1 promoter also changed cell morphology (Fig. $4 \mathrm{~d}^{\prime}$ ) and molecular expression (Fig. $4 \mathrm{~h}, \mathrm{~h}^{\prime}, \mathrm{k}, \mathrm{k}^{\prime}$, $\mathrm{n}, \mathrm{n}^{\prime}, \mathrm{o}$ ), and the position of NeuroD1:Foxg1-overexpressing cells shifted upward (Fig. 4e, $P=0.01$ in BIN3). These results suggested that ectopic Foxg1 expression hampers L4 fate acquisition.

We next asked whether Foxg1 segregates L2/3 and L4 projection neurons upstream of Brn 2 and Ror $\beta$, which exhibit reciprocal expression after E18.5 ${ }^{23}$. Quantitative analysis showed that at E18.5, Foxg1-overexpressing cells showed a significant increase in Brn2 expression (Supplementary Fig. 6i-k), indicating that Foxg1 regulates cell identity upstream of this transcriptional network. Notably, Foxg1-expressing cells were distributed in deeper parts of the CP at this stage (Supplementary Fig. 6g, h).

Previously, Foxg1 was found to promote cortical progenitor cells to enter the cell cycle ${ }^{34}$, as well as control the migration of cortical neurons after exiting the cell cycle ${ }^{35}$. Therefore, changes in laminar identity upon Foxg1 overexpression may be the consequence of cell cycle re-entry or delayed migration. We thus examined the expression of the proliferation markers Ki67 and pH3 1 day after Foxg1 IUE at E14.5. There was no difference in the number of Ki67- or pH3-positive cells between control and Foxg1-overexpressing cells (Supplementary Fig. 6a-e'), implying that the effect of Foxgl overexpression on L4 fate is not due to cell cycle re-entry. We next assessed whether Foxg1 alters cell fate through attenuating cell migration by examining the positions of GFP-positive cells. As mentioned above, at E18.5, the majority of control cells arrived above the Ctip2/Zfpm2-expressing deeplayer cells; however, many Foxg1-overexpressing cells were migrating through Ctip2/Zfpm2 deep-layer cells (Supplementary Fig. 6f, g, h, n, o), raising two possibilities: Foxg1 regulates cell 
a

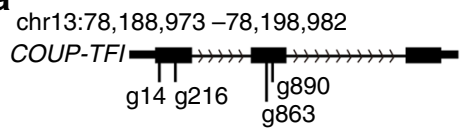

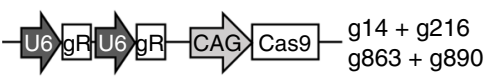

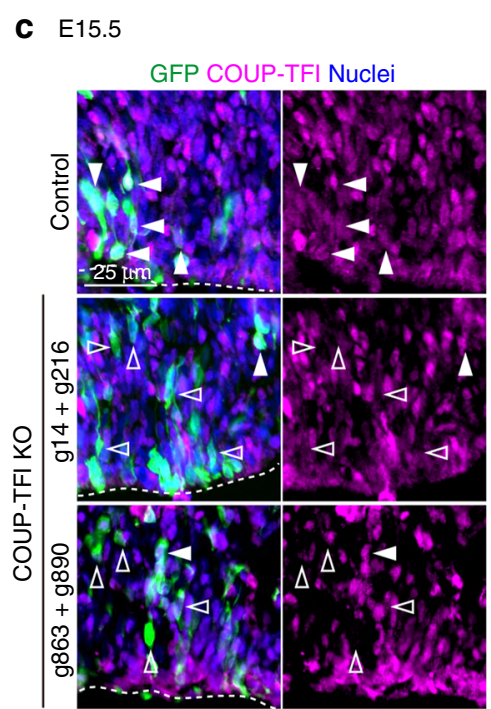

d $\mathrm{E} 15.5$

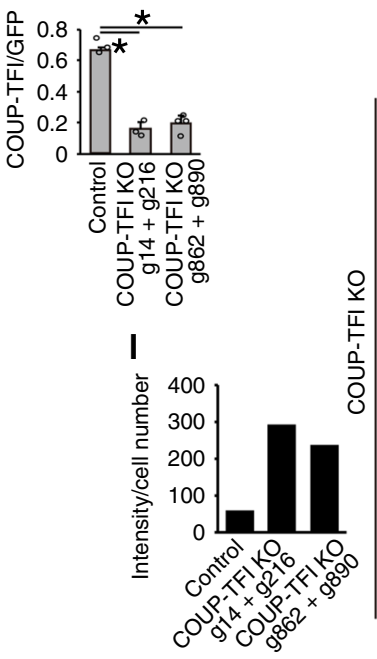

P7 GFP

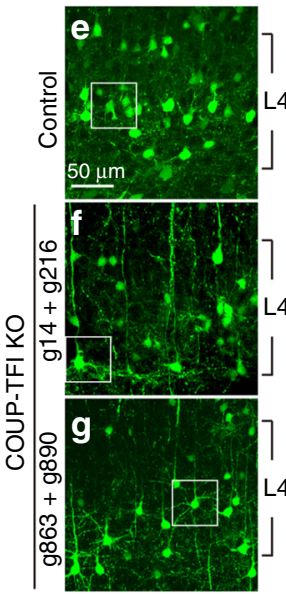

h

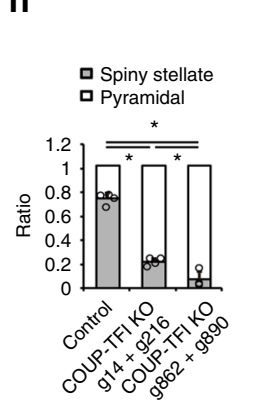

b

pCAG:GFP

$$
\underset{\mathrm{P} 7}{\mathrm{E} 15.5}
$$

Cas9 only or Cas9+gRNAs
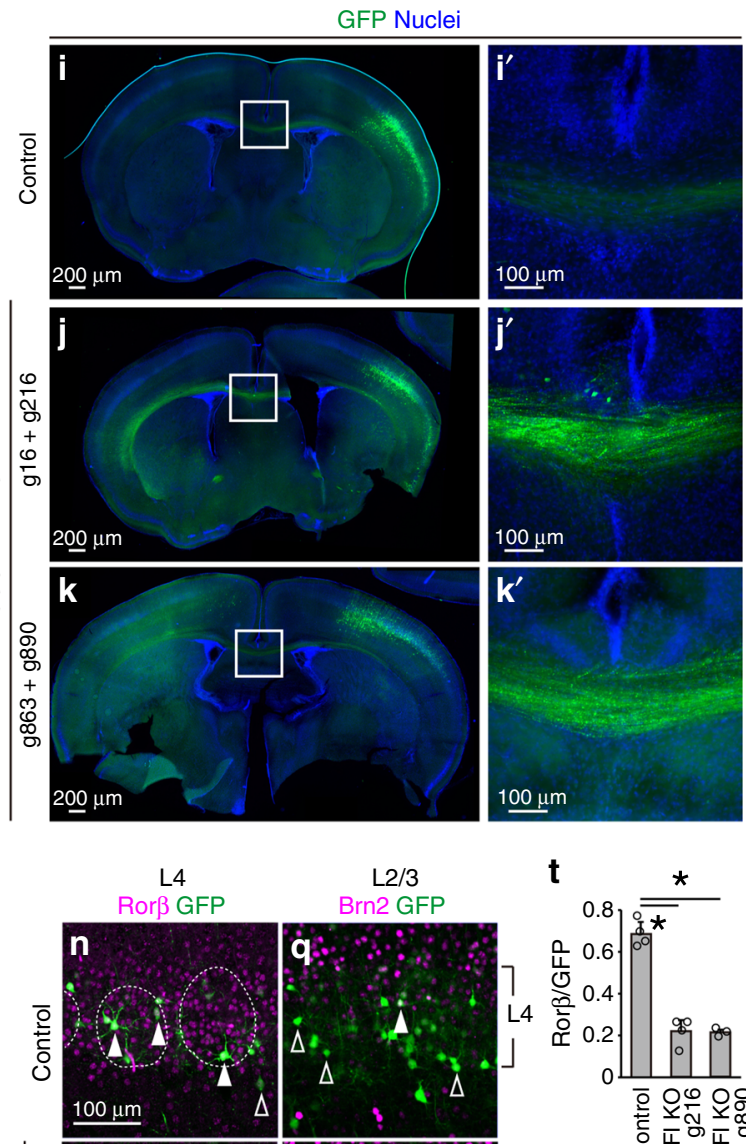

t $0.8 \stackrel{ }{=}$
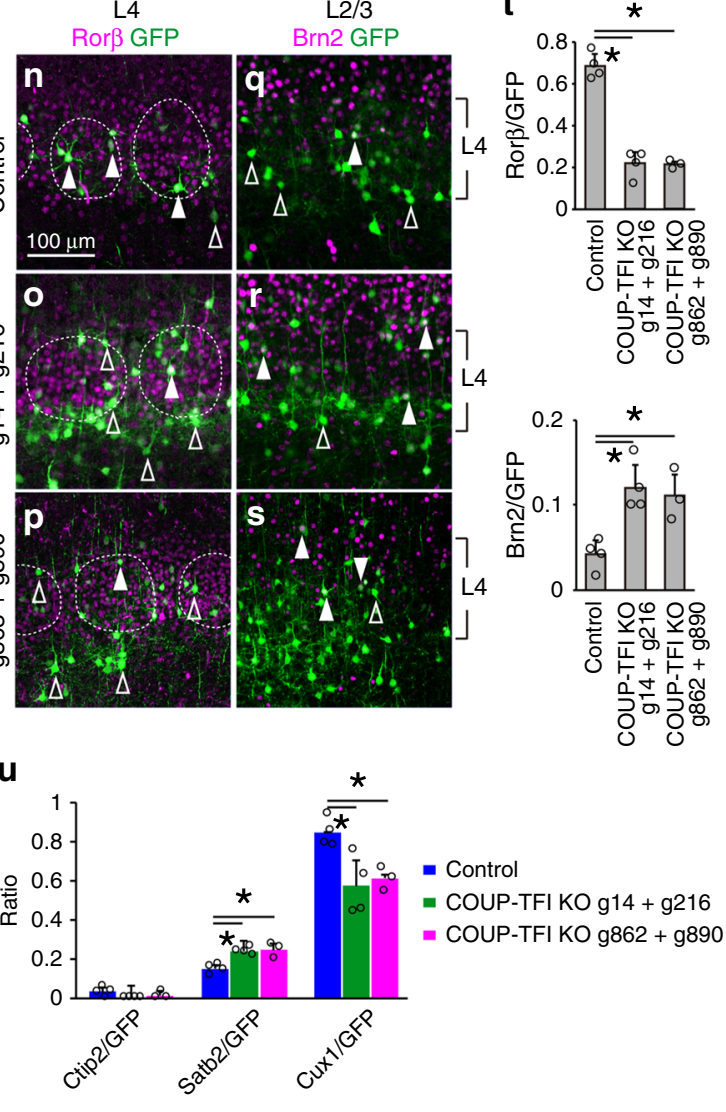

$\mathbf{u}$
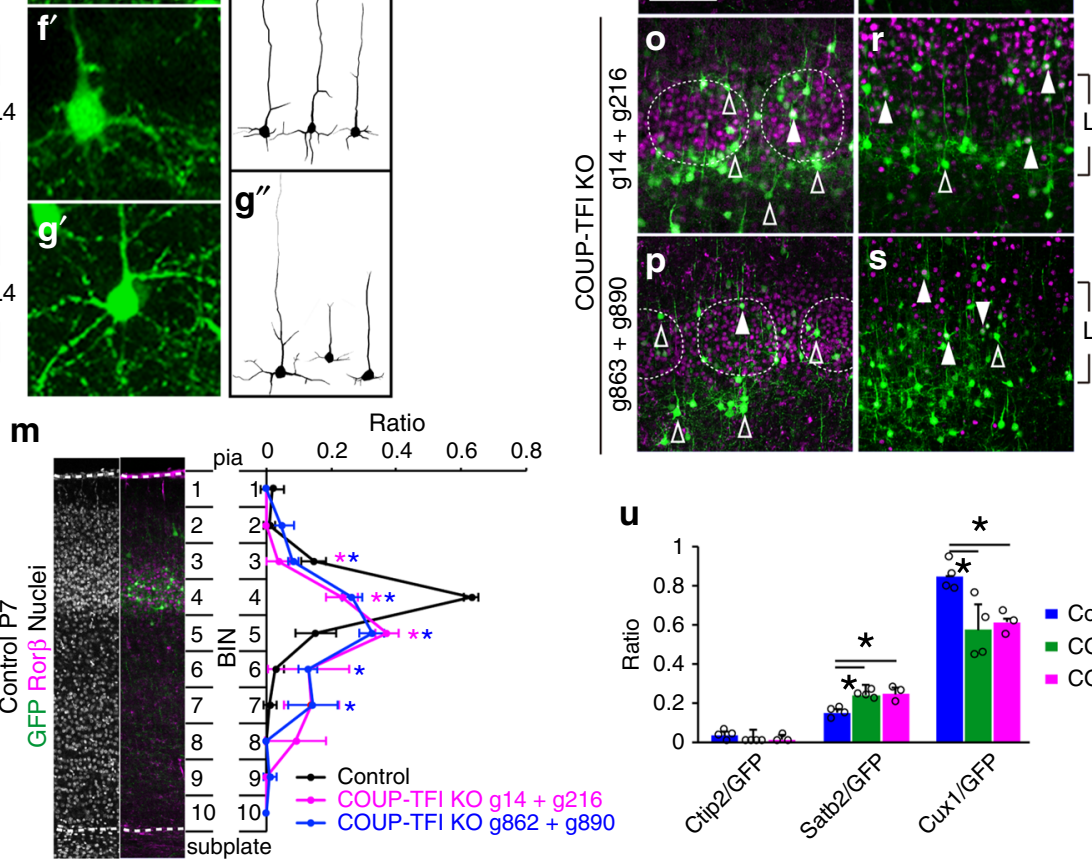

migration, and the altered positioning changed the molecular identity of these cells. Alternatively, Foxg1 defines cell fate, and cells are sorted according to their molecular identity. To differentiate between these possibilities, we tested the consequence of Foxg1 overexpression in deep-layer cells and assessed whether these cells also affect migration and convert to a more upper-layer fate (i.e., L4 cells). We introduced Foxg1 into deeplayer progenitor cells at E12.5 (Fig. 4p). At P7, cells with Foxg1 overexpression were retained in deep layers showing pyramidal morphology similar to that of control cells (Fig. 4q), without increasing the number of Ror $\beta$-positive L4 cells (Fig. 4r-v). Notably, a delayed migration pattern was still observed at 3 days 
Fig. 3 COUP-TFI is required for layer 4 fate acquisition. a Schematic diagram showing four gRNAs designed to target the COUP-TFI gene. gRNAs, g14 and g216, target the first exon of the COUP-TFI gene; gRNAs, g863 and g890, target the second exon of the COUP-TFI gene. b Schematic diagram of in utero electroporation. c GFP (green), COUP-TFI (red) immunohistochemistry, and Hoechst 33342 (blue) of E15.5 cortices. Brains were introduced with pCAG:GFP and UbC:Cas9 (Control) or pCAG:GFP, UbC:Cas9 and gRNAs (COUP-TF1 KO). gRNA combinations are as indicated. Dashed lines indicate the ventricular surface. Closed arrowheads indicate GFP cells with COUP-TFI expression and open arrowheads indicate GFP cells without COUP-TFI expression. $\mathbf{d}$ Quantitative analysis of the percentage ( \pm SEM) of GFP cells that express COUP-TFI. e-g" GFP immunostaining (green) and dendritic reconstruction of GFP cells in P7 cortices. $\mathbf{h}$ Quantitative analysis of the percentage ( \pm SEM) of GFP cells with spiny stellate or pyramidal morphology. $\mathbf{i}-\mathbf{k}^{\prime}$ Immunostaining of GFP (green) and Hoechst 33342 (blue) of P7 cortices. $\mathbf{i}^{\prime}-\mathbf{k}^{\prime}$ demarcate enlarged view of boxed regions shown in (i-k). I Quantitative analysis of the GFP signal intensity in the corpus callosum normalized to GFP-positive cell number. $\mathbf{m}$ Quantitative analysis of the distribution of GFP cells in P7 cortices. Cortical plate is divided into 10 BINs from the pia to the subplate. $\mathbf{n}-\mathbf{s}$ Immunostaining of P7 cortices for GFP with Ror $\beta$ (n-p) or Brn2 (q-s). Dashed lines indicate layer 4 barrel structures. Closed arrowheads indicate GFP cells with Ror $\beta$ or Brn2 expression and open arrowheads indicate GFP cells without Ror $\beta$ or Brn2 expression. $\mathbf{t}$, $\mathbf{u}$ Quantitative analysis of the percentage ( \pm SEM) of GFP cells that express Ror $\beta$ and Brn2 (t), Ctip2, Satb2 or Cux1 (u). * indicates $P$ value $<0.05$ by Student's $t$-test. Source data are provided as a Source Data file

after IUE (Supplementary Fig. 6p-s), while cell fate was not affected (Fig. 4r-v). These results indicated that Foxg1 directly suppresses the fate of L4 cells, thereby causing laminar distribution changes.

FOXG1 binds to COUP-TFI gene locus to suppress layer 4 fate. The effect of Foxg1 overexpression on L4 cells coincided with that of the loss of COUP-TFI in these cells (Fig. 3), suggesting a reciprocal regulation of Foxg1 and COUP-TFI to control L4 fate. We thus asked whether COUP-TFI loss-of-function causes Foxg1 upregulation to attenuate L4 fate or if COUP-TFI is a critical Foxg1 downstream regulator that derepresses L4 fate. Knockout of COUP-TFI using CRISPR/Cas9 did not affect Foxg1 expression 2 days after electroporation (Supplementary Fig. $4 a-d$ ), indicating that Foxg1 upregulation was not the primary cause of the loss of L4 identity upon COUP-TFI deficiency. In contrast, Foxg1 overexpression caused rapid downregulation of COUP-TFI in these cells (Figs. 1d and 2b, c). These results suggested that COUP-TFI is a critical downstream target of Foxg1 that promotes L4 fate.

As we identified a Foxg1-targeted site on the COUP-TFI gene (Fig. 2f-l), we next asked whether elimination of this binding of Foxg1 to COUP-TFI could rescue the loss of L4 fate upon Foxg1 overexpression. We introduced the CRISPR system to disrupt the Foxg1-targeted site together with Foxg1 overexpression into future L4 cells by IUE at E13.75 (Fig. 5a). At P7, cells without Foxg1 overexpression were positioned in L4 and developed spiny stellate morphology (Fig. 5b, c). These cells expressed typical L4 markers, Ror $\beta$ and Cux1 (Fig. 5b, c, f, g, n, q), were in contact with vGlut2-positive thalamic axon terminals (Fig. 5f, g), and did not express Brn2, Satb2, or Ctip2 (Fig. 5b, c, j, k, o, p). Notably, while disruption of the Foxg1-targeted site alone had no significant effect on L4 molecular identity, the position of these cells shifted slightly towards the lower part of L4. In contrast, in line with previous observations, cells with Foxg1 overexpression alone were distributed away from L4 and exhibited pyramidal morphology (Fig. 5d), accompanied by a loss of Ror $\beta$ and Cux1 expression and thalamocortical input (vGlut2) (Fig. 5d, h), but gained Brn2 and Satb2 expression (Fig. 5h, l, o, p). Compared to the introduction of single gRNA, the introduction of two gRNAs rescued the number of $\mathrm{L} 4$ cells, indicating that these cells were refractory to Foxg1 overexpression (Fig. 5e, i, m-q). Cells with two gRNAs and Foxg1 expression were repositioned to L4 and showed spiny stellate morphology (Fig. 5e), showing a L4 molecular identity with Ror $\beta$ and Cux1 but not Brn2 or Satb2 expression (Fig. $5 \mathrm{n}-\mathrm{q})$. Taken together, these results identify COUP-TFI as a critical downstream target of Foxg1 that regulates L4 cell fate acquisition.
COUP-TFI promotes layer 4 fate acquisition. Based on the results above, we hypothesized that the expression of COUP-TFI alone may be sufficient to establish L4 cell fate. We thus introduced COUP-TFI into non-L4 precursor cells and directly assessed whether those cells acquire L4 cell identity. First, we introduced COUP-TFI with GFP at E13.25 to target future L5a cells. At postnatal stages, the majority of control GFP cells were found in L5a and did not express the L4 marker Ror $\beta$ or L5b marker Ctip2 but expressed Satb2 (Supplementary Fig. 7a-d) ${ }^{36,37}$. Thus, we concluded that E13.25 electroporation mainly targets L5a CPNs. We next examined the effect of COUP-TFI GOF in these future L5a cells (Fig. 6a). Consistent with the results in L4 cells, COUP-TFI overexpression did not affect Foxg1 expression 2 days after its introduction (Fig. 6b). At P8, control cells positioned below L4 not contacting vGlut2 thalamocortical axons did not express Ror $\beta$ or Cux1 (Fig. 6c, d, f, h, j, l, n). In contrast, COUP-TFI overexpression increased the number of cells integrated into L4 barrel structures. These cells exhibited spiny stellate-like morphology and expressed L4 markers (Fig. 6c, e, g, i, $\mathrm{k}, \mathrm{m}, \mathrm{n}$ ), and an ectopic barrel was occasionally detected (Fig. 6m, $\mathrm{m}^{\prime}$, compared with the contralateral side in Fig. $\left.6 \mathrm{~m}^{\prime \prime}\right)$. Notably, a few cells outside of barrel structures expressed L4 markers Ror $\beta$ and Cux1 and were enriched with vGlut2 signals (Supplementary Fig. $7 \mathrm{~g}$, i). These results indicated that COUP-TFI can promote future L5a cells to acquire L4 cell fate.

We further tested whether COUP-TFI overexpression could convert L2/3 cells to L4 cells by introducing COUP-TFI at E15.25 (Fig. 6o and Supplementary Fig. 7j-1). At P8, control cells were positioned in L2/3 and expressed Brn2 and Satb2, and devoid of contact with vGlut2-positive thalamic axon terminals (Fig. 6p, q, $\mathrm{s}, \mathrm{u}, \mathrm{w}, \mathrm{y})$. Cells with COUP-TFI overexpression were shifted deeper, and some of them were positioned in L4 and acquired spiny stellate morphology (Fig. 6p). These cells lost the L2/3 molecular identity of Brn2 and Satb2 expression, and a proportion of them expressed Ror $\beta$ and received vGlut2thalamic axon contacts (Fig. 6r, t, v, x, y). Taken together, these results indicate that COUP-TFI GOF can override the intrinsic genetic program to promote L4 fate.

Downregulation of Foxg1 is required for layer 4 competence. Although COUP-TFI overexpression could reprogram non-L4 cells to acquire L4 fate, the efficiency of this reprogramming was higher in L5a cells (Fig. 6a-n) than in L2/3 cells (Fig. 6o-y), implying the influence of other factors. Our results showed that Foxg1 expression in L5a cells was lower than that in L2/3 cells (Fig. $1 \mathrm{j}-\mathrm{j}^{\prime \prime}$ ) and that Foxg1 suppresses COUP-TFI (Fig. 2) and L4 fate (Fig. 4); however, COUP-TFI manipulation did not affect Foxg1 expression (Fig. 6b and Supplementary Fig. 4a-d). These observations suggest that Foxg1 downregulation may be a 
a $\quad$ E13.75

PCAG:GFP

pCAG:empty or CAG:Foxg1

pNeuroD1:Foxg1iresGFP
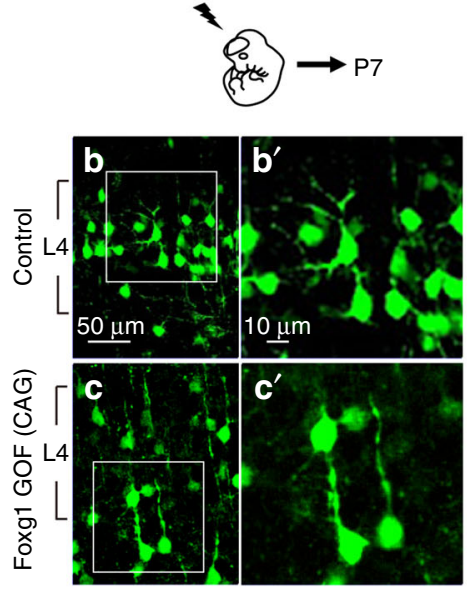

d
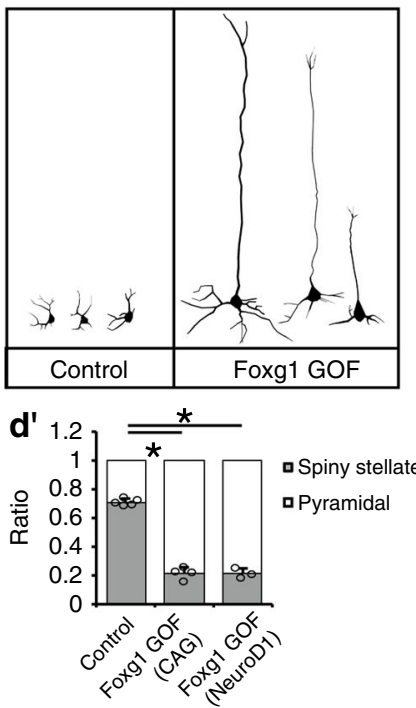

p E12.5

PCAG:GFP

pCAG:empty or pCAG:Foxg1

1
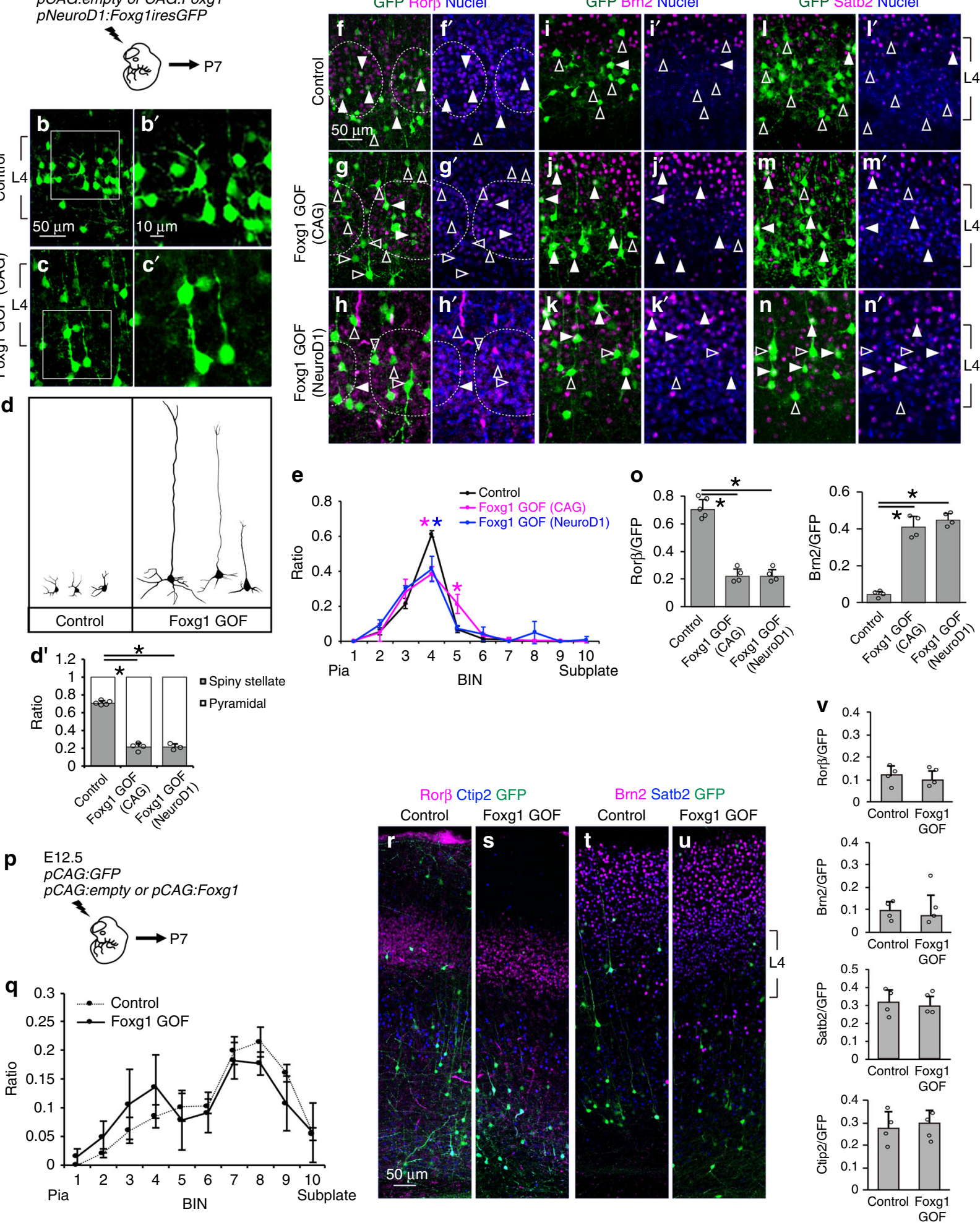

prerequisite to establish L4 cell competence. As Foxg1 have roles in progenitor proliferation ${ }^{34}$, we introduced an inducible in vivo loss-of-function approach using the pInducer tet-inducible knockdown system (Fig. 7a). Two shFoxg1 were designed to knockdown Foxg1 expression, and the efficiency was confirmed by qRT-PCR analysis (Supplementary Fig. 8a, b). These constructs were introduced into the E14.5 neocortex by IUE, and doxycycline was administered from E16.75, at which point the cells have already left the cell cycle (Fig. 7a and Supplementary Fig. 8c, d). At P7, control cells were positioned above L4 and showed pyramidal morphology (Fig. 7b, c, f). In contrast, Foxg1 knockdown cells exhibited a prominent shift into L4 and acquired spiny stellate morphology (Fig. 7b, d-f), with downregulated Brn2 and upregulated Ror $\beta$ expression (Fig. $7 \mathrm{~g}-\mathrm{O}$ ). Notably, some cells 
Fig. 4 Ectopic Foxg1 expression suppresses layer 4 fate. a Schematic diagram of in utero electroporation. Brains were introduced with $p C A G$ :GFP and $p C A G$ : empty (Control) or pCAG:GFP and pCAG:Foxg1 (Foxg1 GOF (CAG)) or pNeuroD1:Foxg1iresGFP (Foxg1 GOF (NeuroD1)). b-d' Immunostaining (b-c'), reconstruction (d), and quantitative analysis of the morphology ( $\mathbf{d}^{\prime}$ ) of GFP cells in P7 cortices. e Quantitative analysis of the distribution of GFP cells in P7 cortices. Cortical plate is divided into 10 BINs from the pia to the subplate. $\mathbf{f}-\mathbf{n}^{\prime}$ Immunostaining of P7 cortices for GFP (green) with Ror $\beta$ (red) (f- $\mathbf{h}^{\prime}$ ), Brn2 (red) (i-k'), Satb2 (red) (I-n'), and Hoechst 33342 (blue). Dashed lines indicate layer 4 barrel structures. Closed arrowheads indicate GFP cells with Ror $\beta$, Brn2, or Satb2 expression and open arrowheads indicate GFP cells without Ror $\beta$, Brn2, or Satb2 expression. o Quantitative analysis of the percentage $( \pm$ SEM) of GFP cells that express Ror $\beta$ or Brn2. p Schematic diagram of in utero electroporation. Brains were introduced with pCAG:GFP and pCAG:empty (Control) or pCAG:GFP and pCAG:Foxg1 (Foxg1 GOF). q Quantitative analysis of the distribution of GFP cells in P7 cortices. Cortical plate is divided into 10 BINs from the pia to the subplate. $\mathbf{r}$-u Immunostaining of P7 cortices for GFP (green) with Ror $\beta$ (red) and Ctip2 (blue) staining ( $\mathbf{r}$, s) or Brn2 (red) and Satb2 (blue) staining $(\mathbf{t}, \mathbf{u})$. v Quantitative analysis of the percentage $( \pm$ SEM) of GFP cells that express Ror $\beta$, Brn2, Ctip2, or Satb2. * indicates $P$ value $<0.05$ by Student's $t$-test. Source data are provided as a Source Data file

a

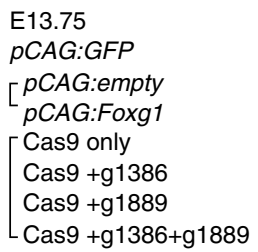

pCAG:GFP

[ $p C A G: e m p t y$

Cas9 only

Cas9 + g1386

Cas $9+\mathrm{g} 1386+\mathrm{g} 1889$
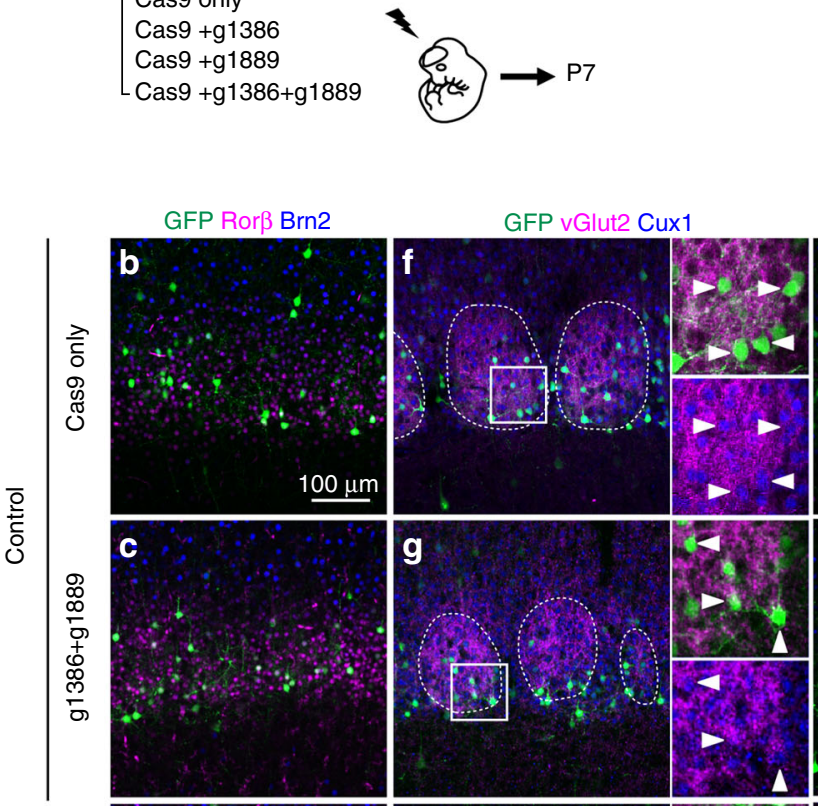

GFP Ctip2 Satb2
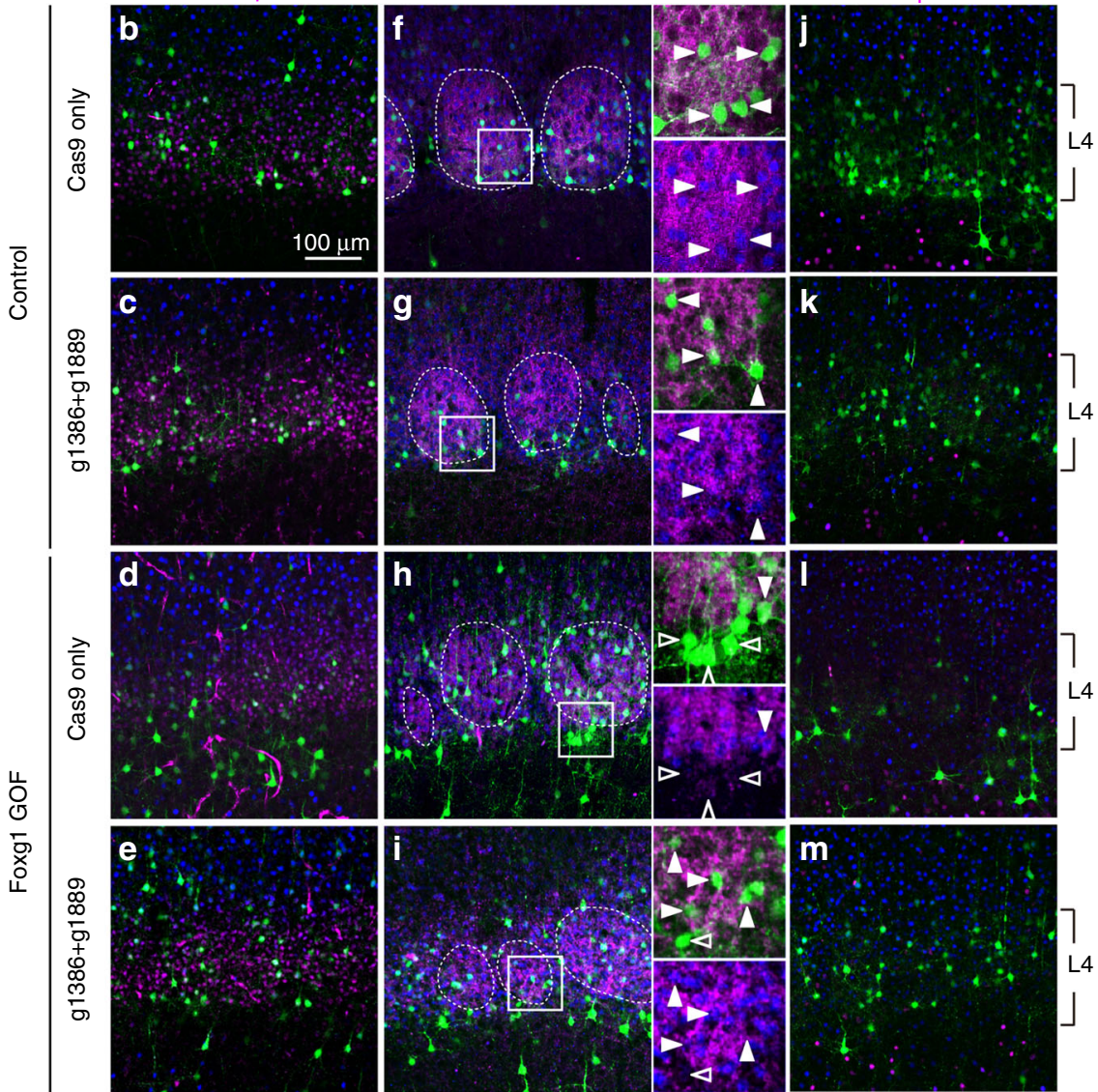
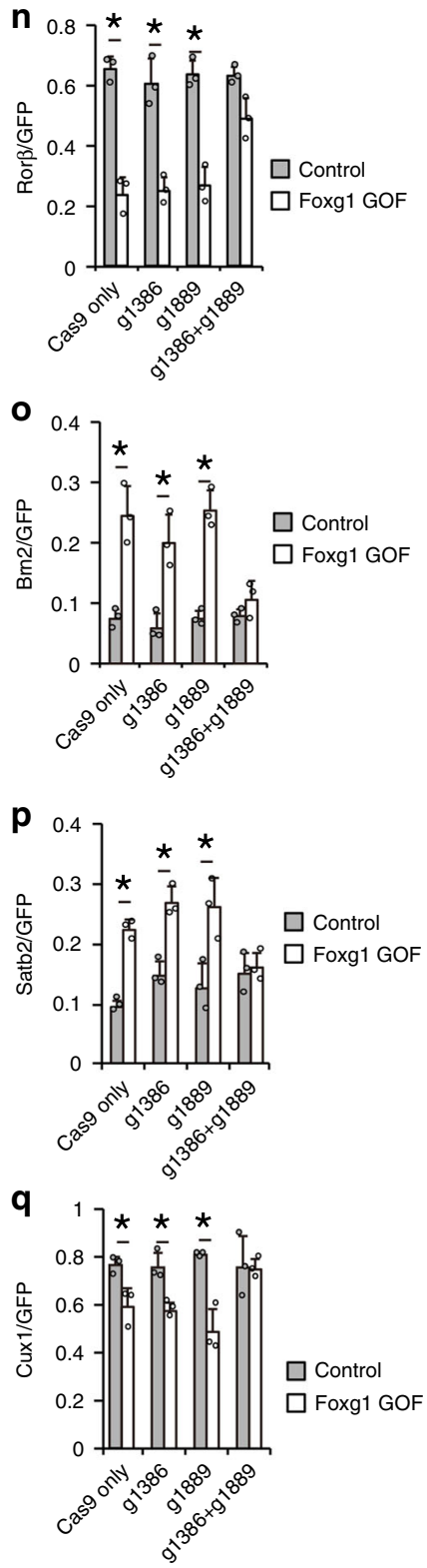

Fig. 5 Restoration of layer 4 fate upon removal of Foxg1 binding to COUP-TFI gene. a Schematic diagram of in utero electroporation. Brains were introduced with pCAG:GFP and pCAG:empty (Control) or pCAG:GFP and pCAG:Foxg1 (Foxg1 GOF). Combinations of gRNAs are as indicated. b-m Immunostaining of P7 cortices for GFP (green) with Ror $\beta$ (red) and Brn2 (blue) (b-e), or vGlut2 (red) and Cux1 (blue) (f-i), or Ctip2 (red) and Satb2 (blue) (j-m). Dashed lines indicate layer 4 barrel structures. Closed arrowheads indicate GFP cells with enriched vGlut2 signal, thalamocortical presynaptic terminals and open arrowheads indicate GFP cells without enriched vGlut2 signal (f-i). $\mathbf{n}-\mathbf{q}$ Quantitative analysis of the percentage $( \pm$ SEM) of GFP cells that express Ror $\beta$ (n), Brn2 (o), Satb2 (p), or Cux1 (q). * indicates $P$ value $<0.05$ by Student's $t$-test. Source data are provided as a Source Data file 
a

E13.25

pCAG:GFP

pCAG:empty or COUP-TFI

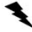

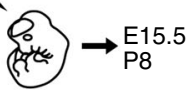

b E15.5, COUP-TFI GOF GFP COUP-TFI

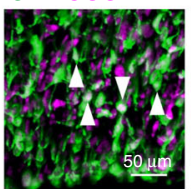

Layer 2-4 GFP Cux1

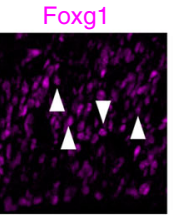

C

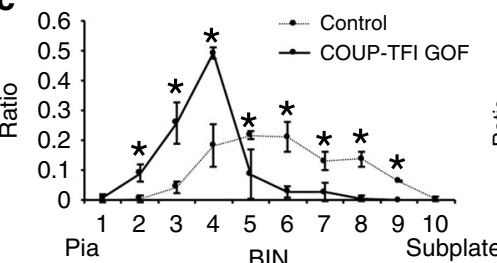

Layer 2/3 Thalamocortical connection
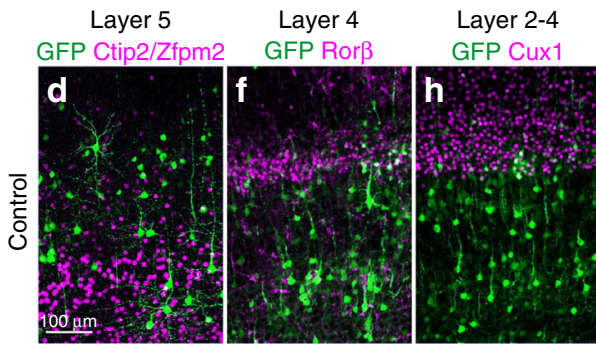
GFP Brn2
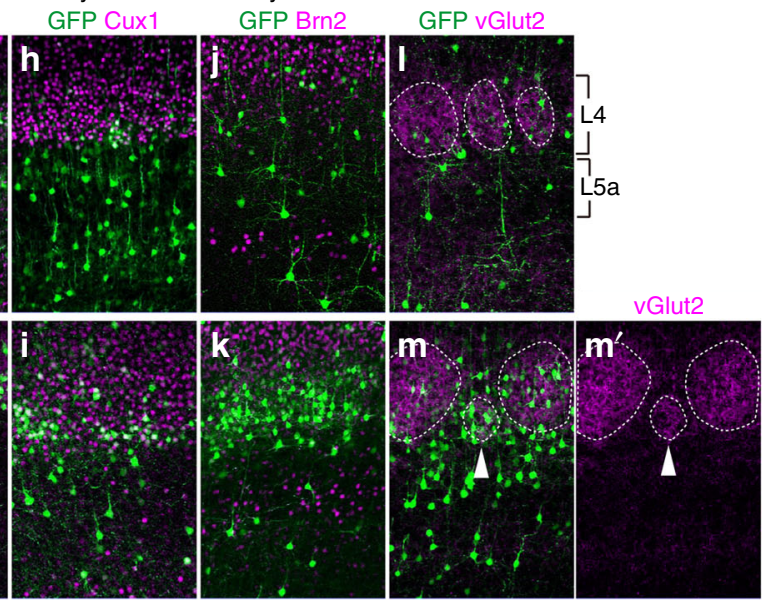

Contralateral
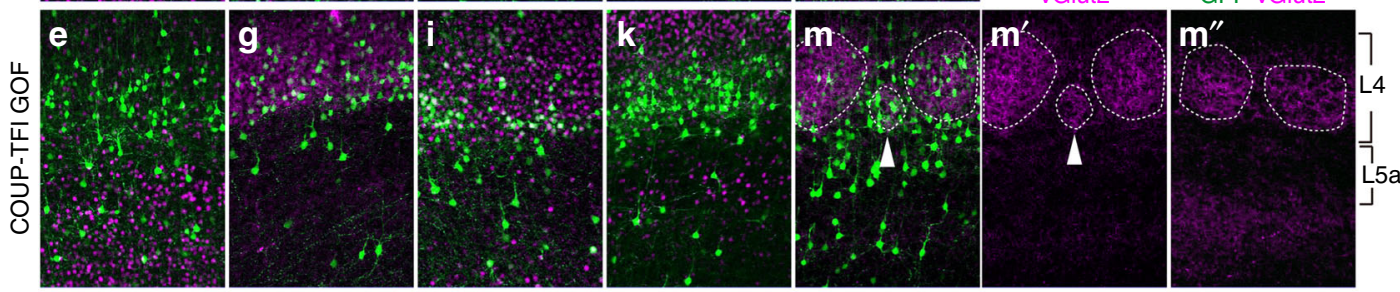

O

E15.25

PCAG:GFP

PCAG:empty or COUP-TFI

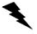

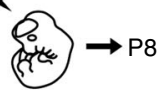

$\mathbf{p}$
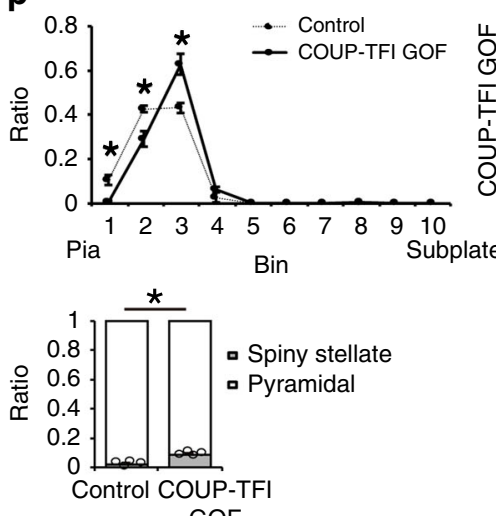

GOF

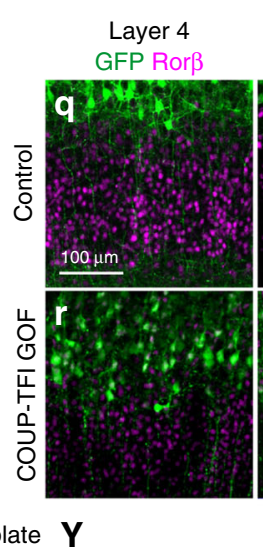

Layer 2/3 GFP Brn2

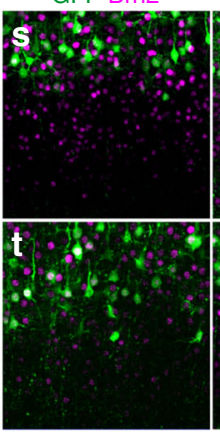

Layer 2/3 Thalamocortical connection GFP vGlut2
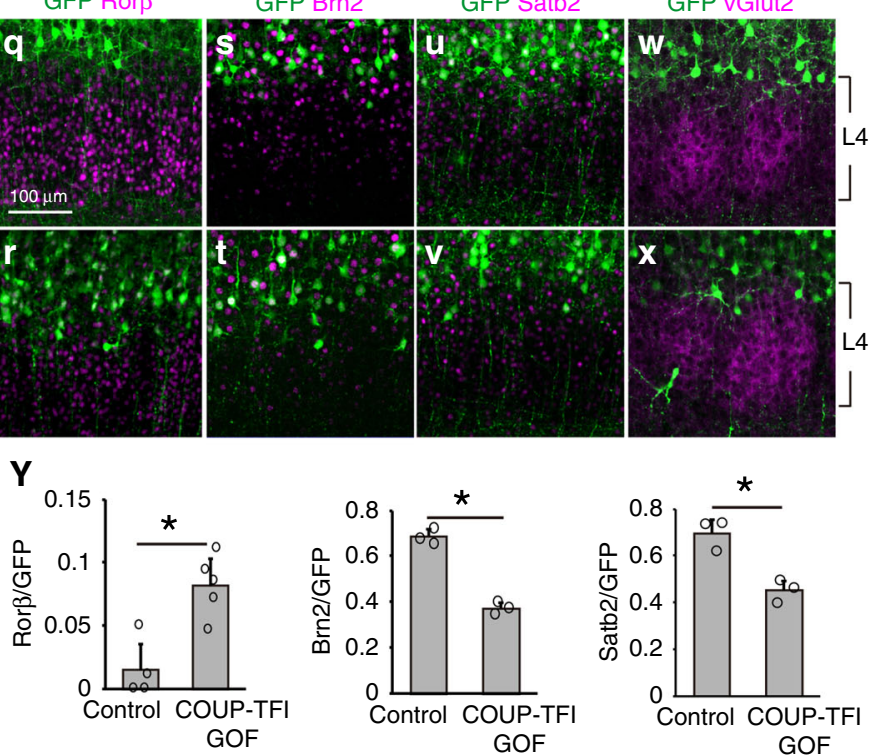

Fig. 6 Ectopic COUP-TFI expression promotes non-layer 4 cells to acquire layer 4 fate. a Schematic diagram of in utero electroporation. Brains were introduced with pCAG:GFP and pCAG:empty (Control) or pCAG:GFP and pCAG:COUP-TFI (COUP-TFI GOF). b Immunostaining of GFP (green), COUP-TFI (red), and Foxg1 (magenta) in E15.5 cortices. Closed arrowheads indicate GFP cells expressing both COUP-TFI and Foxg1. c Quantitative analysis of the distribution of GFP cells in P7 cortices. Cortical plate is divided into $10 \mathrm{BINs}$ from the pia to the subplate. Quantitative analysis of the percentage ( \pm SEM) of GFP cells with spiny stellate or pyramidal morphology. d-m" Double immunohistochemistry of P8 cortices for GFP (green) with Ctip2/Zfpm2 (red) (d, e), $\operatorname{Ror} \beta$ (red) (f, g), Cux1 (red) (h, i), Brn2 (red) (j, k), and vGlut2 (red) (I- $\left.\mathbf{m}^{\prime \prime}\right)$. Dashed lines indicate layer 4 barrel structures. Closed arrowheads indicate ectopic vGlut2-positive thalamocortical presynaptic terminals as compared to the contralateral hemisphere. $\mathbf{n}$ Quantitative analysis of the percentage $( \pm$ SEM) of GFP cells that express Ror $\beta$, Cux1 or Brn2. o Schematic diagram of in utero electroporation. Brains were introduced with $p C A G$ :GFP and $p C A G$ : empty (Control) or pCAG:GFP and pCAG:COUP-TFI (COUP-TFI GOF). p Quantitative analysis of the distribution of GFP cells in P7 cortices. Cortical plate is divided into $10 \mathrm{BINs}$ from the pia to the subplate. Quantitative analysis of the percentage ( \pm SEM) of GFP cells with spiny stellate or pyramidal morphology. $\mathbf{q}-\mathbf{x}$ Double immunohistochemistry for GFP (green) with Ror $\beta$ (red) (q, $\mathbf{r}$ ), Brn2 (red) (s, t), Satb2 (red) (u, v), and vGlut2 (red) (w, x). y Quantitative analysis of the percentage $( \pm$ SEM) of GFP cells that express Ror $\beta, B r n 2$ or Satb2. * indicates $P$ value $<0.05$ by Student's $t$-test. Source data are provided as a Source Data file 
a

E14.5

pCAG:GFP

plnducer10-empty or plnducer10-shFoxg1

(j) $\frac{1 \text { Doxycycline }}{\mathrm{E} 16.75}$ b

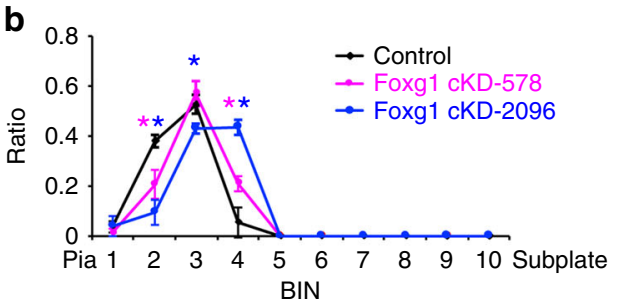

GFP Ror $\beta$ Nuclei
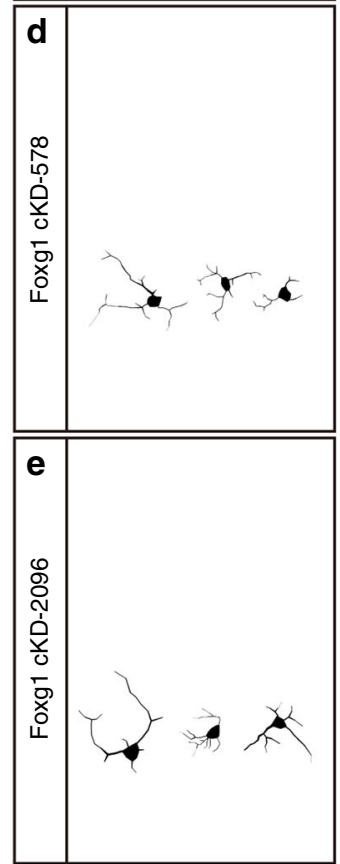

f

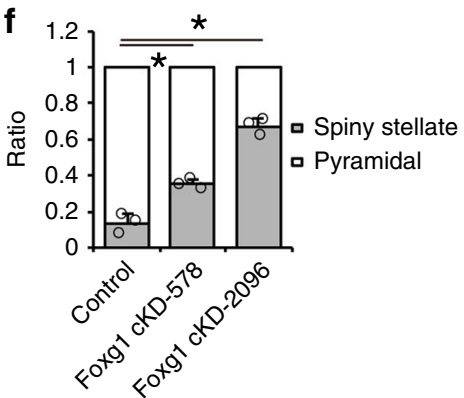

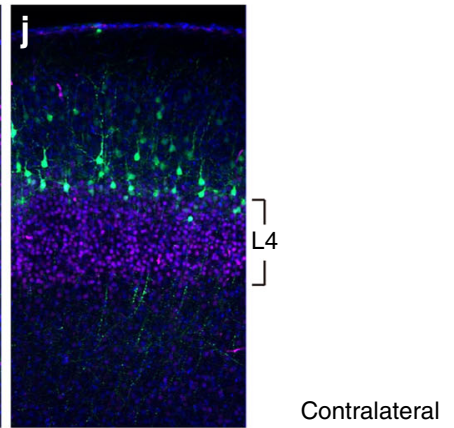

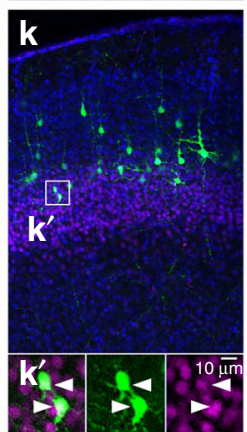

m
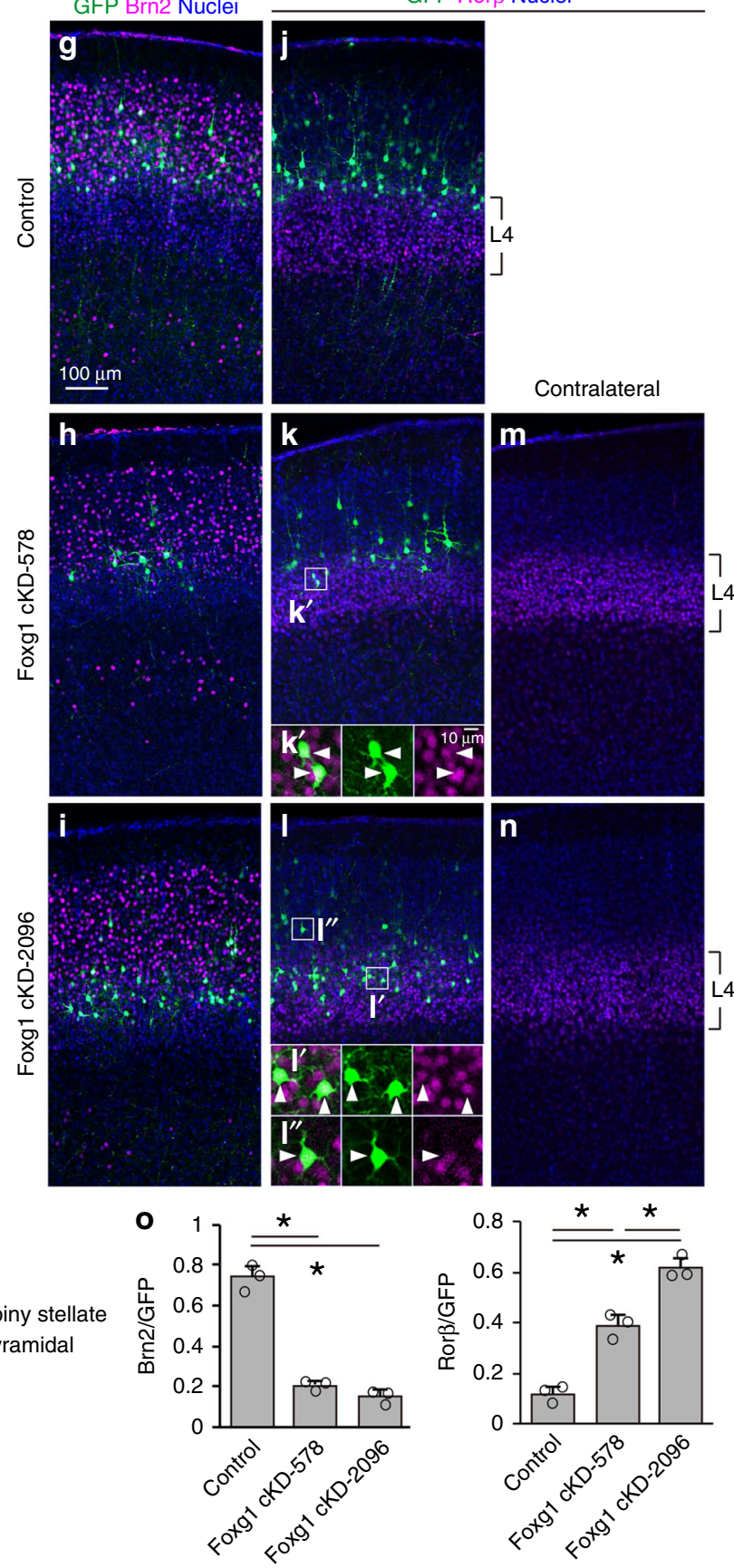

Fig. 7 Conditional suppression of Foxg1 converts layer 2/3 to layer 4 neuron identity. a Schematic diagram of inducible Foxg1 knockdown using the plnducer system. Doxycycline was administered from E16.75 to suppress Foxg1 expression. b Quantitative analysis of the distribution of GFP cells in P7 cortices. Cortical plate is divided into 10 BINs from the pia to the subplate. c-e Representative reconstruction of GFP cells from P7 cortices. $\mathbf{f}$ Quantitative analysis of the percentage ( \pm SEM) of GFP cells with spiny stellate or pyramidal morphology. g-n Double immunohistochemistry for GFP (green) with Brn2 (red) (g-i) or Ror $\beta$ (red) (j-n). o Quantitative analysis of the percentage $\left( \pm\right.$ SEM) of GFP cells that express Brn2 or Ror $\beta .{ }^{*}$ indicates $P$ value $<0.05$ by Student's $t$-test. Source data are provided as a Source Data file 
outside of barrel structures displayed spiny stellate dendritic morphology and expressed the Ror $\beta$ L4 marker (Fig. 7l"). Collectively, these results demonstrate that downregulation of Foxg1 expression is a key requirement to acquire L4 competence.

Egr2 directly suppresses Foxg1 to promote layer 4 fate. Finally, to investigate the upstream molecular mechanism responsible for Foxg1 downregulation in L4 precursor cells, we performed temporal transcriptome analysis to identify genes differentially expressed between earliest L4 and SCPN cells. Previous reports indicated that Neurog2 expression is temporarily upregulated in cells committed to exiting the cell cycle $^{38}$; therefore, we established inducible genetic labeling of temporal precursors by crossing Neurog2 2 reER/+ drivers with Rosa26 $6^{\text {LSL-tdTomato }}$ mice. Upon tamoxifen administration, Cre protein in Neurog2expressing cells induces recombination at the Rosa26 locus, enabling to label the temporal cohorts of cortical neuron precursors with tdTomato (Fig. 8a). 4OHT administration at E13.5 labeled cell population restricted to deep-layer neurons, while administration at E15.25 selectively labeled L4 cells (Fig. 8b and Supplementary Fig. 10). To examine the difference between L4 cells and deep-layer cell transcripts, tdTomato-positive cells from the somatosensory cortex were sorted using a fluorescenceactivated cell sorter (FACS) at 24 and $48 \mathrm{~h}$ after $4 \mathrm{OHT}$ administration and subjected to RNA sequencing (Fig. 8b). Significant differential expression genes among samples $(P$ value $<0.05)$ showed early differences in deep-layer precursors and L4 precursors (Fig. 8c). Notably, the deep-layer projection neuronspecific genes Ctip2, Fezf2, Tbr1, Sox5, and Foxg1 were enriched in E13.5-labeled cells, whereas the L4 marker gene Pcdh20 was enriched in L4 precursor cells (Fig. 8c). Using this platform, we performed in silico analysis to identify upstream signals. Ingenuity Pathway Analysis found that TGF $\beta$ and Fgf signaling were more activated in L4 precursor cells than in deep-layer precursor cells (Fig. 8d). Notably, activated TGF $\beta$ and Fgf signaling were detected at both 24 and $48 \mathrm{~h}$ after $4 \mathrm{OHT}$ administration. To investigate the direct regulator of Foxg1, we submitted $1.3 \mathrm{~kb}$ upstream of the Foxg1 gene to TFBIND software (http://tfbind.hgc.jp) to search for TF binding sites (Fig. 8e, f). Apart from common transcriptional regulators, a panel of TF binding sites were found on the Foxg1 upstream region. Interestingly, we identified multiple binding sites of the Egr family within $200 \mathrm{bp}$ (Fig. 8f). Egr1, a previously reported activitydependent gene ${ }^{39}$, was expressed in cortical neurons (Fig. 8g, $g^{\prime}$ ). Egr2, a downstream target of TGF $\beta$ signaling ${ }^{40}$, was also detected in the intermediate zone and CP (Fig. $8 \mathrm{~h}, \mathrm{~h}^{\prime}$ ), and was upregulated in early L4 precursor cells (Fig. 8c). Together, these results suggested that Egr genes control Foxg1 expression to regulate L4 cell competence. To directly assess this hypothesis, we introduced Egr1 or Egr2 into the E14.5 neocortex by IUE (Fig. 8i). At E16.5, Egr2 GOF reduced Foxg1 expression, whereas Egr1 had a milder impact on Foxg1 expression (Fig. 8j-m). At P7, in controls, cells contributed to L2/3 (Fig. 8n-p), and Egr1 overexpression had no effect on L4 molecular identity (Fig. 8q-s, w, x). In contrast, Egr2 overexpression caused a similar effect as conditional Foxg1 knockdown (Fig. 7), where cells shifted to L4 and acquired a Ror $\beta$-positive L4 molecular identity (Fig. 8t-x).

We further assessed the Egr regulation on L4 fate using loss-offunction studies (Fig. 9a-1). We introduced the CRISPR/ Cas9 system to disrupt either the Egr1 or Egr2 gene in future L4 cells using IUE at E13.75, and analyzed the consequence at P7 (Fig. 9a-1). Loss of either Egr1 or Egr2 expression caused failure of cells to gain L4 fate and cells exhibited a prominent shift in position (Fig. 9b), acquired pyramidal morphology, lost Ror $\beta$ expression, and gained Brn2 expression (Fig. 9d-1). Notably,
Egr1-deficient cells apparently shifted towards deeper regions, while Egr2-deficient cells were excluded from barrels (Fig. 9b), similar to COUP-TFI-deficient cells (Fig. 3) and Foxg1overexpressing cells (Fig. 4a-o). Furthermore, some Egr1deficient cells gained Ctip2 expression, which was not observed in Egr2-deficient cells (Fig. 91). Together with the Egr1 and Egr2 GOF studies (Fig. 8i-x), these results suggest that Egr1 and Egr2 have differential roles in L4 development.

As Egr is predicted to target the Foxg1 promoter to suppress its expression, we further examined whether the presumptive Egrtargeted Foxg1 promoter region is responsible for Egr-Foxg1 regulation in L4 fate selection (Figs. $8 \mathrm{e}$ and $9 \mathrm{~m}$ ). We disrupted the Egr-targeted Foxg1 promoter region in future L4 cells using CRISPR/Cas9 (Fig. 9m-v). At E15.5, prominent Foxg1 upregulation in cells introduced with gRNAs were observed, indicating that this region is capable of controlling Foxg1 expression (Fig. 9n). At P7, cells with gRNAs were excluded from L4 and showed pyramidal morphology (Fig. 9o, o', q, s, u) and expressed less Ror $\beta$ (Fig. 9q, q', v) and more Satb2 (Fig. 9s, s', v) compared to control cells. The effects of disrupting the Egr-targeted Foxg1 promoter region on attenuating L4 fate were similar to those of disrupting Egr2 expression (Fig. 9a-1), Foxg1 overexpression (Fig. 4a-o) and COUP-TFI loss-of-function (Fig. 3). Conversely, Egr2 overexpression (Fig. 8i-x), conditional Foxg1 disruption (Fig. 7) and COUP-TFI overexpression studies (Fig. 6) resulted in L4 fate acquisition. Taken together, these results identify an EgrFoxg1-COUP-TFI regulatory network in the preselection of projection neuron subtypes (Fig. 10).

\section{Discussion}

L4 neurons play fundamental roles in the cerebral cortex, acting as a gateway for peripheral inputs to initiate sensory information processing in higher order brain regions ${ }^{12}$. Such a unique function has evolved increased susceptibility of L4 cells to extrinsic cues than distally projecting CFuPNs and CPNs. Therefore, their developmental mechanisms has required the consideration of both cell-autonomous and non-cell autonomous mechanisms. We provide lines of evidence that demonstrate that L4 cell competence is established through an Egr-Foxg1-COUP-TFI network to confer their unique molecular and hodological properties.

In the neocortex, the tight correlation between cell fate and laminar identity ${ }^{41,42}$ has raised a question concerning the causal relationship between cell positioning and fate specification of projection neuron subtypes. In this study, removal of COUP-TFI or the acquisition of Foxg1 in L4 precursors resulted in delayed cell migration (Supplementary Figs. $4 \mathrm{e}-\mathrm{h}$ and $6 \mathrm{f}-\mathrm{h}$ ); however, these neurons were shifted either superficially or deeper relative to the somatosensory barrels and lost L4 molecular identity (Figs. 3 and $4 a-0$ ). These results indicate that the fate switch is the primary cause underlying laminar identity changes and not the converse. In support of this notion, our results show that Foxg1 overexpression in deep-layer cells does not result in these cells acquiring a L4 cell fate (Fig. $4 \mathrm{p}-\mathrm{v}$ ), consistent with the aberrant lamination but correct molecular specification seen in the Reln mutant cortex ${ }^{9}$.

Currently, the control of the timing of sequential projection subtype specification is still under debate $14,16,17,43,44$. Our study suggests that the fate of $\mathrm{L} 4$ neurons is determined relatively early during development. As Foxg1 GOF using the CAG or NeuroD1 promoter blocked L4 fate acquisition (Fig. 4a-o) and COUP-TFI GOF by the NeuroD1 promoter failed to promote L4 fate (Supplementary Fig. 9), the time window in which the fate of L4 neurons is specified appears to be restricted. Based on the position of E14.5-targeted cells at the timing of doxycycline treatment 
a

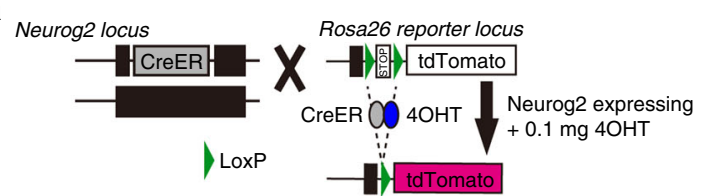

C $\quad \frac{E 13.5}{+24 h+48 h+24 h+48 h+72 h}$

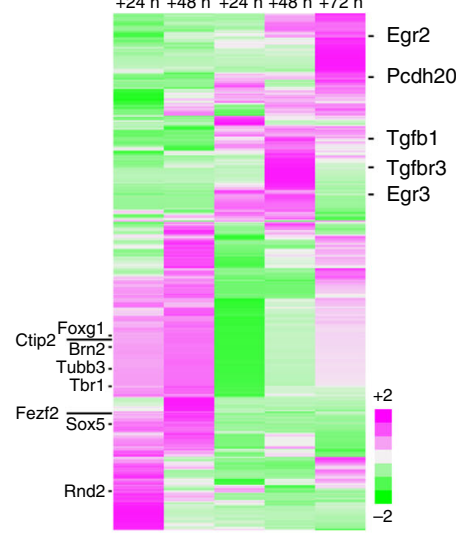

E16.5

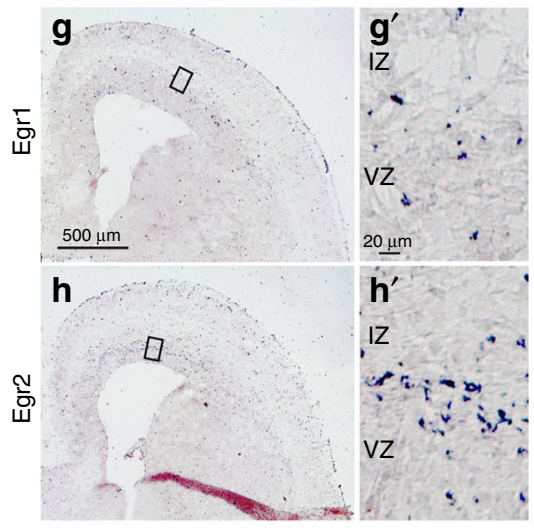

m

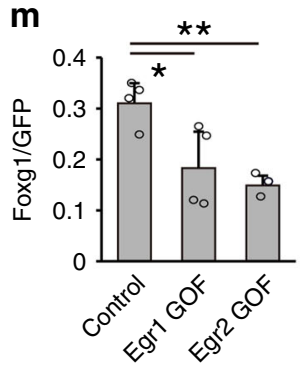

\section{b}

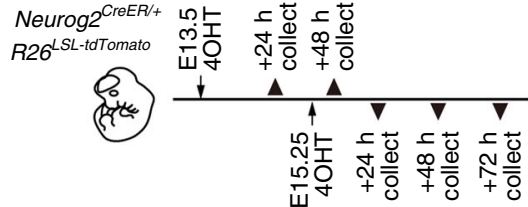

d

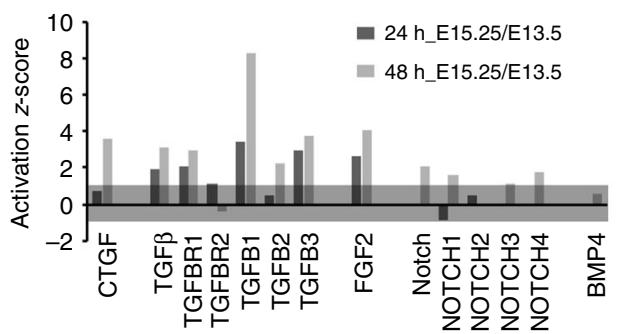

i E14.5 pCAG:GFP pCAG:empty or Egr1 or Egr2

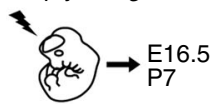

E16.5
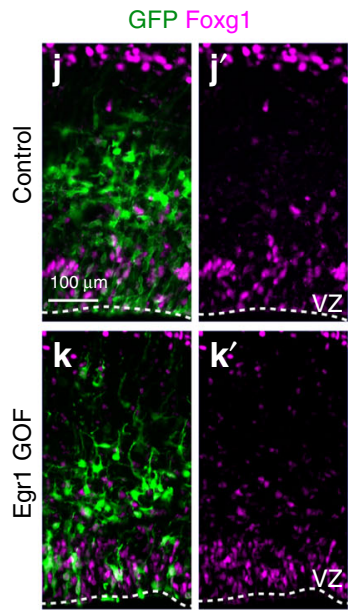

$\mathbf{k}^{\prime}$

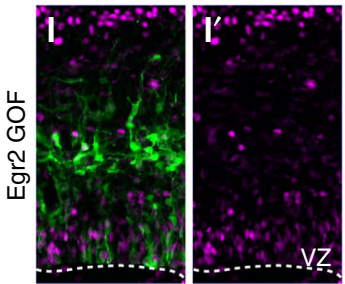

P7 e

Foxg1 locus

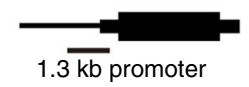

f

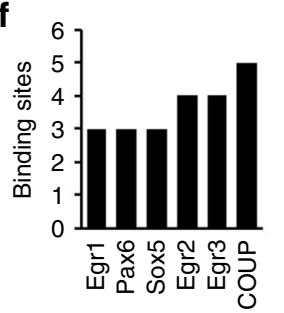

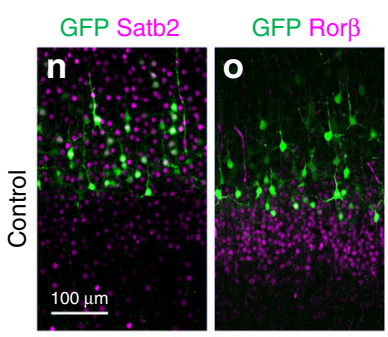
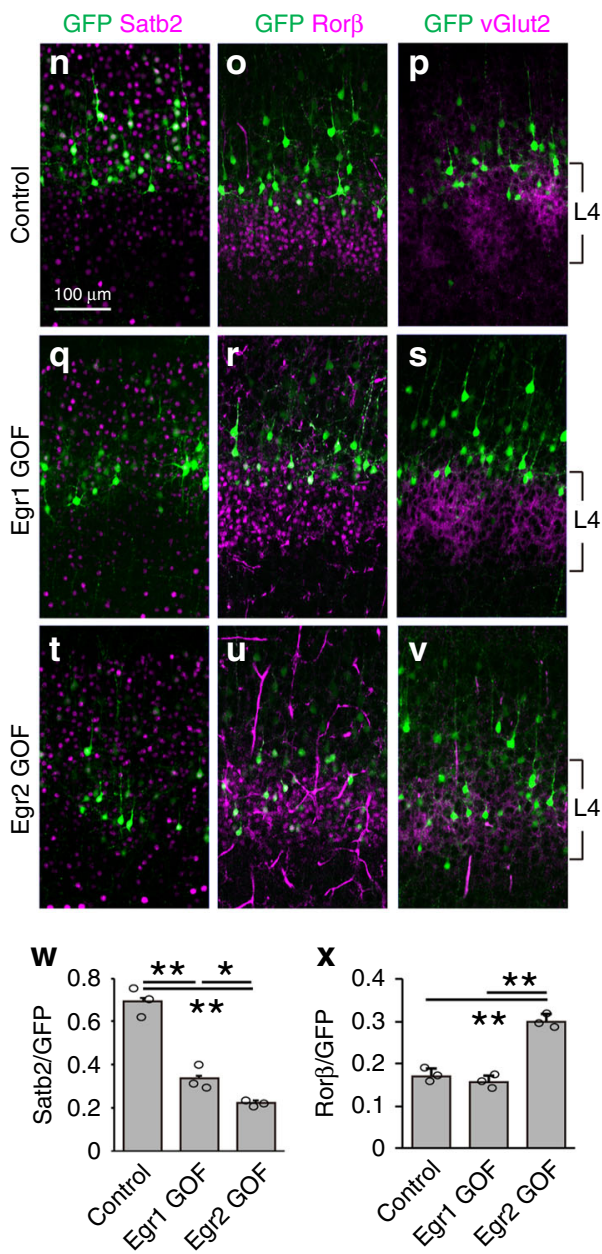

(i.e., Foxg1 conditional knockdown, Supplementary Fig. 8c, d) and the spatiotemporal expression dynamics of Egr2 (Fig. 8h, h'), Foxg1 and COUP-TFI (Fig. 1e-e $\mathrm{e}^{\prime \prime}$ ), together with the reported NeuroD1 expression ${ }^{45}$, Neurog 2 expression ${ }^{46}$, and transcriptomic profile during neurogenesis (Supplementary Fig. 11) ${ }^{47}$, the timing of high-Egr2 and low-Foxg1 expression coincides with Tbr2 expression prior to NeuroD1 peak expression (Supplementary
Fig. 11). This Egr2-Foxg1 regulation causes subsequent COUPTFI upregulation, which may explain why COUP-TFI GOF by the NeuroD1 promoter is too late to promote L4 fate. Taken together, the results indicate that short- and long-range projection neuron fate commences at the early postmitotic stage, where suppression of Foxg1 by Egr2 results in COUP-TFI upregulation to specify L4 fate. We identified TGF $\beta$ signaling as a Foxg1 
Fig. 8 Identification of Foxg1 upstream regulators in projection subtype selection. a Schematic diagram of temporal precursor cell labeling using Neurog2 2 reER/+ and R26LL-tdTomato mediated genetic recombination upon 4OHT administration. b Schematic diagram of sample collection. Neurog2 2 reER/+; R26LSL-tdTomato mice were administered with $4 \mathrm{OHT}$ at E13.5 or E15.25, and brains were collected at the indicated time points. c Heatmap representing hierarchical clustering using the complete linkage with Euclidean distance. Datasets were obtained from two independent analyses normalized to Z-score and shown in average. Genes with significant $P$ value less than 0.01 by differential expression on edgeR and with enriched expression in E13.5 or E15.5 $4 \mathrm{OHT}$ administration were selected. d Ingenuity Pathway Analysis comparing cells from E15.25 and E13.5 4OHT treatment at 24 and $48 \mathrm{~h}$. Candidate upstream regulators related to intercellular signaling pathway with $P$ value less than $10^{-3}$ are shown. Positive and negative values of $Z$-score represent activated or repressed state, respectively. Threshold of $Z$-score $=1$ and -1 is marked in gray. e Schematic diagram indicating the $1.3 \mathrm{~kb}$ Foxg1 promoter positioned at chr12:50,483,149-50,585,435 on the UCSC genome (NCBI37/mm9). $\mathbf{f}$ Quantitative analysis of transcription factors with multiple predicted binding sites on the same strand of the Foxg1 gene (http://tfbind.hgc.jp). g-h' Expression of Egr1 and Egr2 mRNA by in situ hybridization in E16.5 cortices. i Schematic diagram of in utero electroporation. Brains were introduced with pCAG:GFP and pCAG:empty (Control) or pCAG:GFP and pCAG:Egr1 (Egr1 GOF), or pCAG:GFP and pCAG:Egr2 (Egr2 GOF). Dashed lines indicate the ventricular surface. j-I' Double immunohistochemistry of GFP (green) and Foxg1 (red) in E16.5 cortices. $\mathbf{m}$ Quantitative analysis of the percentage ( \pm SEM) of GFP cells that express Foxg1. $\mathbf{n}-\mathbf{v}$ Immunostaining of P7 cortices with GFP (green), Satb2 (red) (n, $\mathbf{q}, \mathbf{t}), \operatorname{Ror} \beta$ (red) $(\mathbf{o}, \mathbf{r}, \mathbf{u})$, and vGlut2 (red) (p, $\mathbf{s}, \mathbf{v})$ antibodies. $\mathbf{w}, \mathbf{x}$ Quantitative analysis of the percentage $( \pm$ SEM) of GFP cells that express Satb2 $(\mathbf{w})$ or Ror $\beta(\mathbf{x})$. VZ ventricular zone, IZ intermediate zone. * indicates $P$ value $<0.05$ and ${ }^{\star *}$ indicates $P$ value $<0.01$ by Student's $t$-test. Source data are provided as a Source Data file

upstream regulator of its repression in L4 cell development (Fig. 8). These results are consistent with a previous reported function of TGF $\beta$ in cortical development, where intraventricular TGF $\beta$ application suppressed Foxg1 expression in cortical progenitor cells ${ }^{48}$, implying a conserved regulation of the TGF $\beta$ Foxg1-COUP-TFI pathway in cortical cell fate specification.

The complementary expression of COUP-TFI and Foxg1 in the developing neocortex is indicative of the reciprocal regulation between these two factors (Figs. 1 and 2). Our results demonstrated that the direct binding of Foxg1 to the COUP-TFI regulatory element is responsible for the suppression of L4 fate (Figs. 3-5), and that Foxg1 downregulation is a prerequisite for L4 cell fate determination upon COUP-TFI GOF (Fig. 7). Previous studies have shown that misexpression of COUP-TFI by the D6 promoter suppresses Fezf2 expression ${ }^{49}$ and that Fezf2 expression is derepressed by Foxg1 through suppressing Tbr ${ }^{21}$. As Fezf2 is the central mediator of SCPN differentiation ${ }^{22}$, collectively, these results reveal for the first time the transcriptional regulatory network that segregates distal and local projection neurons mediated through Foxg1-COUP-TFI-Fezf2.

While the conclusions drawn from this study rely on null function experiments, the fine-tuning of projection cell types may also require dose-dependent control of TFs. Indeed, the complementary roles of COUP-TFI and Foxg1 in brain development are consistent with the appearance of the opposed phenotype observed in human pathogenic variants ${ }^{50-53}$. These studies imply that the imbalance between motor outputs and sensory inputs through dysregulated projection subtype selection may lead to altered sensory processing in congenital neurodevelopmental disorders.

\section{Methods}

Animals. Two independent conditional Foxg1 knockout mouse lines were utilized in this study. For the tet-inducible Foxg1 mouse line, Foxg1 ${ }^{\text {tetOFoxg1 }}$ conditional mutant mice were generated by crossing Foxg1 $1^{\text {tTA/+ }}$ mice with Foxgl ${ }^{\text {lacZ/+}}$; tetOFoxg1-IRESlac $Z$ double-heterozygous mice as previously described ${ }^{20}$. Foxg $1^{t T A /+}$ heterozygous littermates were used as controls. To control Foxg1 expression in vivo, $200 \mu \mathrm{g}$ doxycycline in $250 \mu \mathrm{L} 5 \%$ sucrose was administered orally on the 1st day, and $200 \mathrm{mg} / \mathrm{L}$ doxycycline in $5 \%$ sucrose was provided in drinking water during treatment. For conditional Foxg1 removal in the dorsal telencephalon (Foxg1 cKO), Emx Cre/+ Foxg $^{f /+}$; R26R CAG-FRTstop-EGFP/+ $(R C E-$ $F R T)$ mice were crossed with Foxgfl/fl; RCE-FRT mice 35,54 , and $E m \times 1^{C r e /+}$; Foxgfl/+; $R C E-F R T$ mice were used as controls. Foxg1 null mutants (Foxg1 KO) were generated by intercrossing Foxgl lacz/+ heterozygous mice ${ }^{55}$.

To isolate temporal cortical neuronal precursors, we crossed Neurog2 $2^{C r e E R /+}$ heterozygous mice ${ }^{56}$ with $R 26^{C A G-L S L-t d T o m a t o}$ mice $^{57}$ (Fig. 8a). (Z)-4-

Hydroxytamoxifen solution (4OHT, H7904, Sigma) was prepared in $100 \%$ ethanol and further diluted with corn oil to reach a final concentration of $1 \mathrm{mg} / \mathrm{ml}$. A total of $0.1 \mathrm{mg} / 100 \mu \mathrm{L} 4 \mathrm{OHT}$ per mouse was administered by intraperitoneal injection. The day of vaginal plug detection was designated as embryonic day (E) 0.5 . Both males and females were used in the experiments. Mice were housed in the Animal Housing Facility of the RIKEN Center for Developmental Biology (Kobe, Japan) and Waseda University Animal Facility (Tokyo, Japan) following the institutional guidelines.

Constructs. For Foxg1 GOF studies, pCAG:Foxg1 and pNeuroD1:FoxgliresGFP constructs were used as previously described ${ }^{20,35}$. For GOF, COUP-TFI, the Egrl and Egr2 genes were amplified using primers (COUP-TFI forward: GCTCCCTGGGCC CAAAGAT; COUP-TFI_reverse: TCTCCTGGTTTGCAGCTCAG; Egr1_forward: ACCACCCAACATCAGTTCTC; Egr1_reverse: AAGAAAGCAAAGGGAGAGGC; Egr2_forward: TGTGCGAGGAGCAAATGATG; Egr2_reverse: CACCGTGAGATG AAGCTCT) from cDNA prepared from E17.5 mouse neocortex and subcloned into the $p C A G G S$ vector backbone ( $p C A G$ :COUP-TFI, $p C A G: E g r 1$, and $p C A G: E g r 2$ ) or the $p$ NeuroD1:iresGFP backbone ( $p$ NeuroD1:COUP-TFIiresGFP). To disrupt the Foxg1-targeted region (PBS1 KO, Figs. 2i-1 and 5), COUP-TFI gene (COUP-TF1 KO, Fig. 3), Egr1 gene (Egr1 KO, Fig. 9a-l), Egr2 gene (Egr2 KO, Fig. 9a-1), or Egrtargeted region (Fig. $9 \mathrm{~m}-\mathrm{v}$ ), the CRISPR/Cas9 system was applied ${ }^{58}$ using the following designed sgRNAs (http://chopchop.cbu.uib.no) ${ }^{59}$ : two sgRNAs for PBS1 KO: g1386: TCTACGCGGCGTACTTGCCTCGG, g1889: TCACCAGCTTCGGAAACA TCGGG; two pairs of sgRNAs for COUP-TFI KO: g14: TGCTGGCTCTGGCCTG AACCGGG, g216: TAGCAGCTGGCGAGATCCGCAGG for the first exon and g863: GGTCCATGAAGGCCACGACGCGG, g890: CCACCTGTTCCTGAAAGAT GCGG for the second exon; two sgRNAs for Egr1: GGCGATCGCAGGACTCGACAGGG and TGGCTTCCCGTCGCCGTCAGTGG; two sgRNAs for Egr2: ATCCGTAATTTTACTCTGGGGGG and GGAGCGAAGCTACTCGGATACGG; and two sgRNAs for the Egr-targeted region: g61: GTAGCCGCGATCGATCATC CTGG, g352: TTCACAGCCGAGCTCGCCGCGGG. sgRNA was cloned into the pX330 backbone according to the instruction manual (Addgene). For conditional Foxg1 knockdown experiments (Foxg1 cKD, Fig. 7), inducible shRNA constructs were named according to the position of the shRNA targets: Foxgl cKD-578 targeting the coding sequence: CCTGACGCTCAATGGCATCTA; Foxg1 cKD-2096 targeting the 3' UTR: GCCTTCAGTTTGTGTTGTGTA. Hairpin oligonucleotides were annealed in vitro using annealing buffer containing $10 \mathrm{mM}$ Tris- $\mathrm{HCl} \mathrm{pH} \mathrm{7.5,}$ $50 \mathrm{mM} \mathrm{NaCl}$ and $1 \mathrm{mM}$ EDTA with $50 \mu \mathrm{M}$ forward and reverse oligonucleotides. The following thermal cycle was used: $95^{\circ} \mathrm{C}$ for $2 \mathrm{~min}$, followed by cooling to $20^{\circ} \mathrm{C}$ by decreasing the temperature by $5^{\circ} \mathrm{C} / 10 \mathrm{~min}$. Annealed oligonucleotides were inserted into the EcoRI- and XhoI-digested pInducer10-mir-RUP-PheS vector (Addgene).

In utero electroporation. DNA solutions were prepared with $0.1 \%$ Fast Green (Wako) in Hanks' balanced salt solution (HBSS) at the following concentrations: for Foxg1 GOF, $0.5 \mu \mathrm{g} / \mu \mathrm{L} p C A G$ :GFP and $1 \mu \mathrm{g} / \mu \mathrm{L} p C A G$ :empty or $p C A G$ :Foxg1; for Foxg1 GOF by NeuroD1 promoter, $1 \mu \mathrm{g} / \mu \mathrm{L}$ pNeuroD1:Foxg1iresGFP; for COUP-TFI GOF: $1 \mu \mathrm{g} / \mu \mathrm{L} p C A G: G F P$ and $1 \mu \mathrm{g} / \mu \mathrm{L} p C A G$ :empty or $p C A G$ :COUPTFI; for COUP-TFI GOF by NeuroD1 promoter, $1 \mu \mathrm{g} / \mu \mathrm{L} p N e u r o D 1: C O U P-$ TFIiresGFP; for COUP-TFI KO, PBS1 KO, Egr1 KO, Egr2 KO, and Egr-targeted region $\mathrm{KO}, 1 \mu \mathrm{g} / \mu \mathrm{L} p C A G: G F P$ and $1 \mu \mathrm{g} / \mu \mathrm{L} p U b C: C a s 9$ or $p U b C: C a s 9$ with gRNAs; for Foxg1 GOF with PBS1 KO: $1 \mu \mathrm{g} / \mu \mathrm{L} p C A G: G F P, 1 \mu \mathrm{g} / \mu \mathrm{L} p C A G$ :empty or $p C A G$ :Foxg1, and $1 \mu \mathrm{g} / \mu \mathrm{L} p U b C: C a s 9$ or $p U b C$ :Cas 9 with gRNAs. Pregnant wild-type ICR mice were deeply anesthetized by intraperitoneal injection using Nembutal sodium solution (Lundbeck) or three types of mixed anesthetic agents containing $5.0 \mathrm{mg} / \mathrm{kg}$ butorphanol (Meiji Seika), $4.0 \mathrm{mg} / \mathrm{kg}$ midazolam (SAN$\mathrm{DOZ}$ ), and $0.75 \mathrm{mg} / \mathrm{kg}$ medetomidine (NIPPON ZENYAKU KOGYO CO. ${ }^{60}$. After uterine horn exposure, the DNA solution was transuterally injected into the lateral ventricles with a $0.6-\mathrm{mm}$ inner-diameter capillary (GD-1, NARISHIGE) pulled by the PC-100 capillary puller (NARISHIGE). The embryo stage 
a

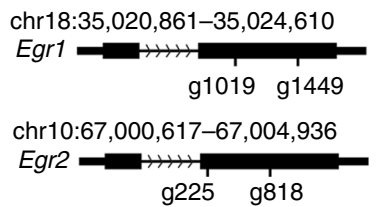

E13.75

pCAG:GFP

Cas9 oniy or Cas9+gRNAs

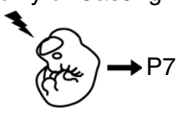

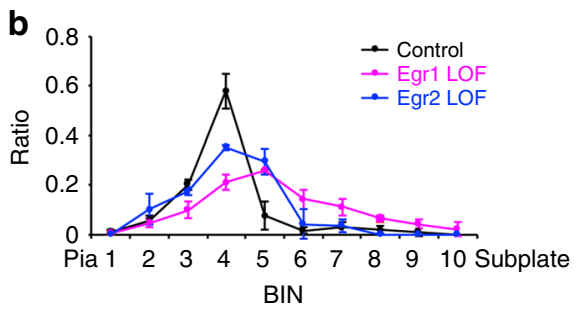

GFP Ctip2 Nuclei
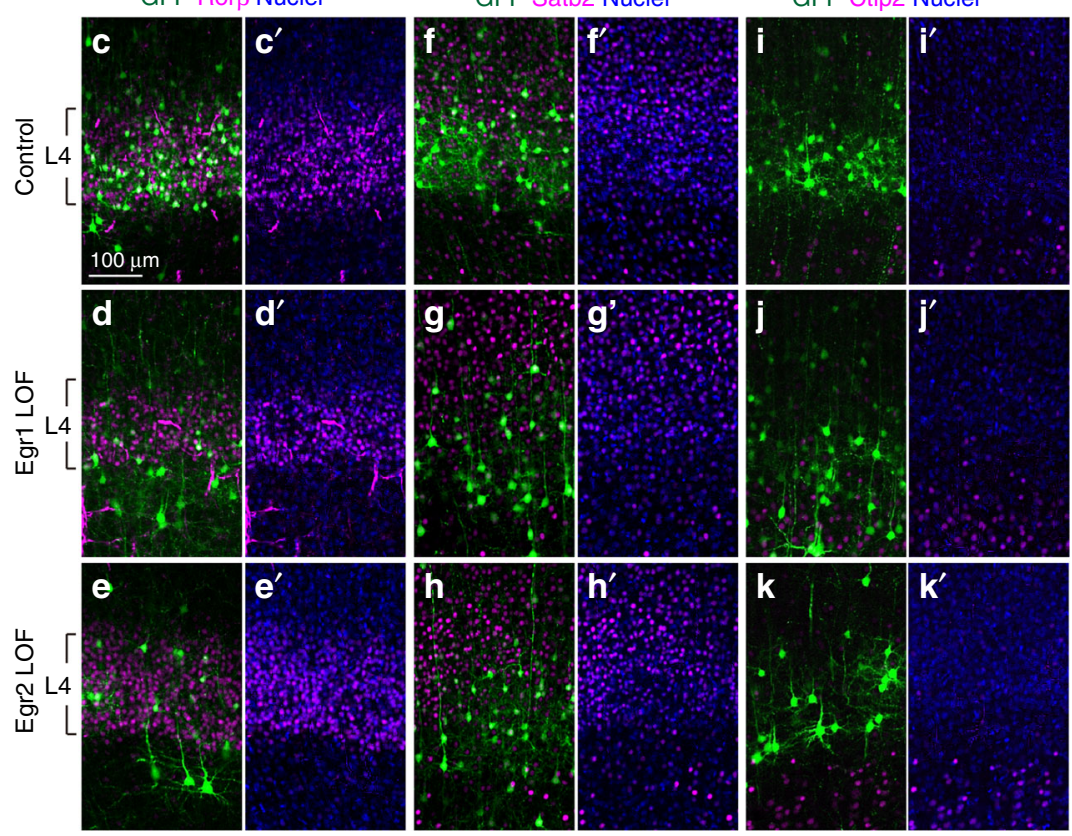

I
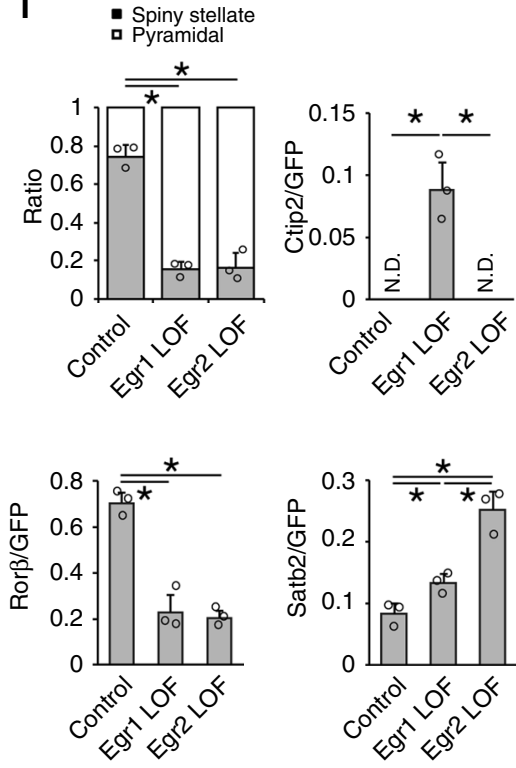

(

n E15.5

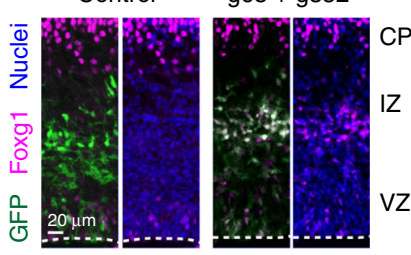

GFP Ctip2 Nuclei

P7

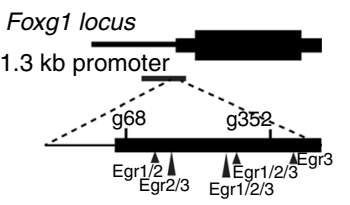

E13.75

pCAG:GFP

Cas9 only or Cas9+gRNAs

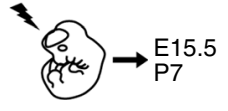

GFP Satb2 Nucle

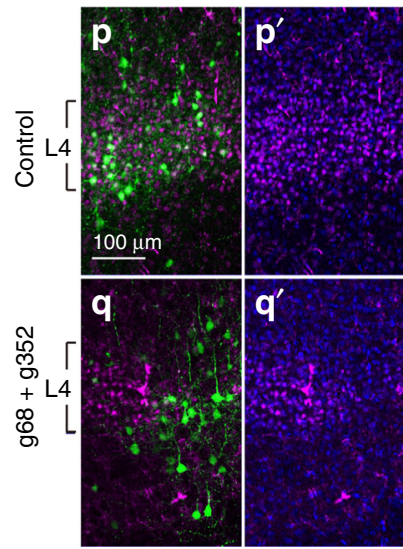

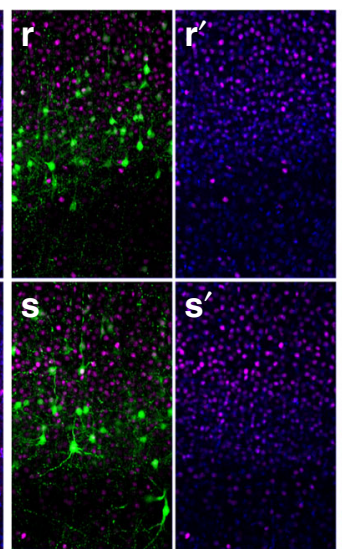

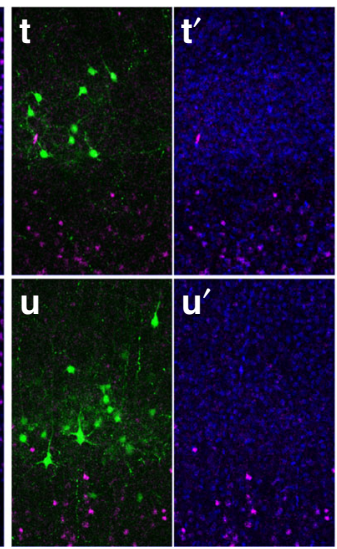

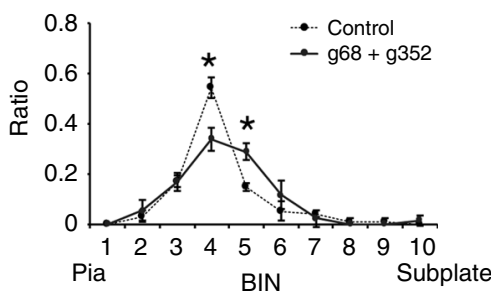
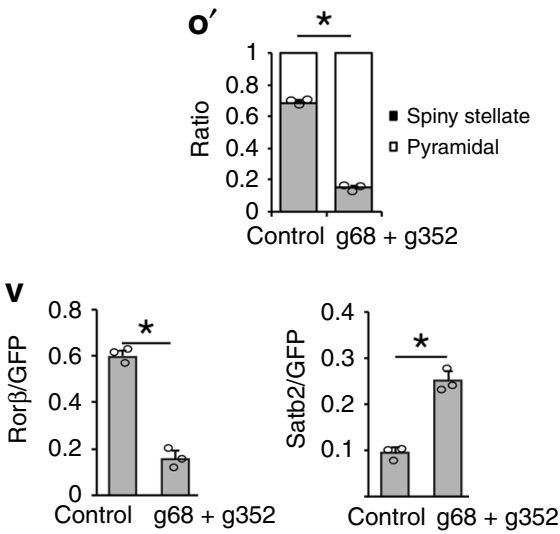

and corresponding voltage were as follows: E12.5 and E13.25: $30 \mathrm{~V}$; E13.75: $33 \mathrm{~V}$; E13.75 and E14.5: $35 \mathrm{~V}$, with a 50-ms on/950-ms off period with a total of 4 pulses using a platinum electrode (CUY650P5; Nepagene). Primary somatosensory area was targeted throughout this study. After electroporation, the abdominal wall and skin were sutured, and the mice were kept at $37^{\circ} \mathrm{C}$ until recovery.
Immunohistochemistry and image acquisition. Embryonic brains up to E17.5 were collected directly, and brains from embryos $>$ E17.5 and postnatal stages were perfused with $\mathrm{PBS}$ and $4 \%$ paraformaldehyde (PFA) prior to fixation. Embryonic brains were fixed with $4 \%$ PFA at $4{ }^{\circ} \mathrm{C}$ for $1 \mathrm{~h}$, and postnatal brains were fixed with $2 \%$ PFA overnight. For cryosection, brains were immersed in $30 \%$ sucrose-PBS at $4{ }^{\circ} \mathrm{C}$ overnight and embedded in Tissue-Tek O.C.T compound (Sakura). 12- $\mu \mathrm{m}$ - 
Fig. 9 Removal of Egr target site derepresses Foxg1 and suppresses layer 4 fate. a Schematic diagram of gRNA design and in utero electroporation. b Quantitative analysis of the distribution of GFP cells in P7 cortices. Cortical plate is divided into 10 BINs from the pia to the subplate. c-k' Immunohistochemistry of P7 cortices

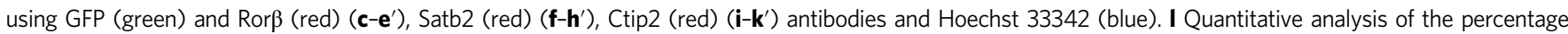
$( \pm$ SEM) of GFP cells with spiny stellate or pyramidal morphology and the percentage ( \pm SEM) of GFP cells that express Ror $\beta$ or Satb2, or Ctip2. $\mathbf{m}$ Schematic diagram of gRNA design and in utero electroporation. Brains were introduced with pCAG:GFP and Cas9 only (Control) or pCAG:GFP and Cas9 with gRNAs (g68 + g352). n Immunostaining of E15.5 cortices for GFP (green) with Foxg1 (red) staining and Hoechst 33342 (blue). Dashed lines indicate the ventricular surface. VZ ventricular zone, IZ intermediate zone, CP cortical plate. $\mathbf{0}, \mathbf{o}^{\prime}$ Quantitative analysis of the distribution of GFP cells in P7 cortices. Cortical plate is divided into $10 \mathrm{BINs}$ from the pia to the subplate (o). Quantitative analysis of the percentage ( \pm SEM) of GFP cells with spiny stellate or pyramidal morphology

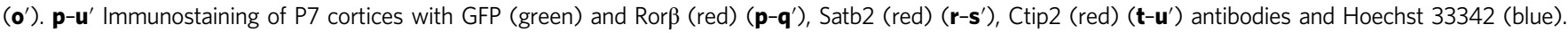
$\mathbf{v}$ Quantitative analysis of the percentage $( \pm$ SEM) of GFP cells that express Ror $\beta$ or Satb2. * indicates $P$ value $<0.05$ by Student's $t$-test. Source data are provided as a Source Data file

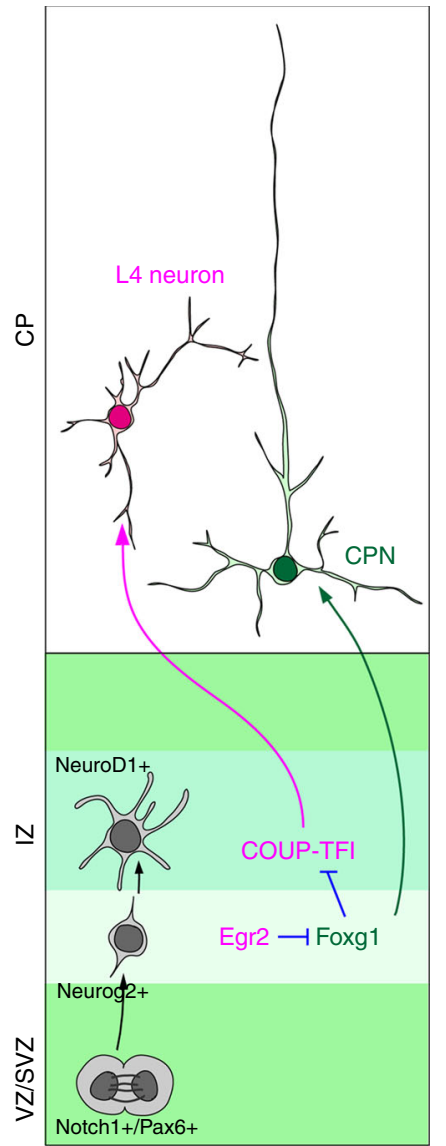

Fig. 10 Proposed model for short- and long-range projection neuron selection. After the cell cycle exit, Egr2 is activated in early postmitotic neurons and suppresses Foxg1 expression in the lower intermediate zone. This downregulation of Foxg1 derepresses COUP-TFI in postmitotic neurons and confers short-range projection neuron identity. VZ/SVZ ventricular zone/subventricular zone, IZ intermediate zone, CP cortical plate

thick sections were collected using a cryostat (Microm HM550; Carl Zeiss) and stored at $-80^{\circ} \mathrm{C}$ until analysis. For floating sections, postnatal brains were embedded in $1.4 \%$ agarose and $1 \%$ gelatin in PBS and sectioned using a vibratome slicer $(150 \mu \mathrm{m})(\mathrm{NLS}-\mathrm{AT}$, Dosaka). Floating sections were processed immediately for immunostaining. For immunostaining on cryosections, blocking solution containing $10 \%$ normal donkey serum and $0.05 \%$ Triton X-100 in PBS was applied at room temperature for $1 \mathrm{~h}$, and primary antibodies were applied in $1 \%$ normal donkey serum and $0.05 \%$ Triton X-100 in PBS at $4{ }^{\circ} \mathrm{C}$ overnight. After the tissue was washed, secondary antibodies were applied in $0.05 \%$ Triton X-100 in PBS at room temperature for $30 \mathrm{~min}$. Nuclei were stained with Hoechst 33342 (ThermoFisher). For immunostaining on floating sections, blocking solution containing $10 \%$ normal donkey serum, $0.3 \%$ Triton X-100, and $0.1 \%$ sodium azide in PBS was applied at $4{ }^{\circ} \mathrm{C}$ overnight. Primary antibodies were applied in $1 \%$ normal donkey serum, $0.3 \%$ Triton $\mathrm{X}-100$, and $0.1 \%$ sodium azide in PBS at $4{ }^{\circ} \mathrm{C}$ for 3 overnights.
After the tissue was washed, secondary antibodies were applied in $1 \%$ normal donkey serum, $0.3 \%$ Triton X-100, and $0.1 \%$ sodium azide in PBS at $4{ }^{\circ} \mathrm{C}$ overnight. Nuclei were stained with Hoechst 33342 (ThermoFisher). Sections were mounted with SlowFade Gold Antifade Mountant (ThermoFisher). Images were acquired using an Axio Imager Z1 fluorescence microscope (Carl Zeiss), FV1000 confocal microscope (Olympus), LSM780 and LSM800 confocal microscope (Carl Zeiss). For dendritic reconstruction, the semiautomated tracing software Neurolucida was applied for images captured using an LSM800 confocal microscope. The following primary antibodies were used: rat anti-GFP (1:500, NACALAI), chicken anti-GFP (1:500, Abcam), rabbit anti-BF1/Foxg1 (1:500, TaKaRa), mouse anti-COUP-TFI (1:200, Perseus Proteomics), rat anti-Ctip2 (1:500, Abcam), mouse anti-Ror $\beta$ (1:100, Perseus Proteomics), rabbit anti-Cux1/CDP (1:100, Santa Cruz), goat antiBrn2 (1:100, Santa Cruz), rabbit anti-Satb2 (1:500, Abcam), rabbit anti-Zfpm2 (1:100, Santa Cruz), guinea pig anti-vGlut2 (1:500, Millipore), rabbit anti-DsRed (1:500, Clontech), mouse anti-Ki67 (1:100, BD), and rabbit anti-pH3 (1:200, Millipore). Secondary antibodies conjugated with Alexa Fluor dye were used at 1:500 dilutions (ThermoFisher), except donkey anti-chicken-488 was used at a 1:200 dilution (Jackson ImmunoResearch).

In situ hybridization. Digoxigenin (DIG)-labeled Foxg1 antisense probes were prepared as previously described ${ }^{61}$. Egr1 and Egr2 antisense probes were prepared from FANTOM clones (I730052D02 for Egrl and E430002H10 for Egr2) and used as previously described ${ }^{62}$. Briefly, samples were pretreated and hybridized with probes $(100 \mathrm{ng} / \mathrm{ml})$ at $55^{\circ} \mathrm{C}$ overnight. The anti-DIG antibody (1:500, Roche) was applied at $4{ }^{\circ} \mathrm{C}$ overnight. Foxg1 signals were developed using Texas-Red streptavidin (1:500, Vector) at room temperature for $2 \mathrm{~h}$. After the tissue was washed with PBS, immunostaining was performed from a blocking step with $10 \%$ normal donkey serum and $0.05 \%$ Triton X-100 in PBS. The primary antibody, mouse antiCOUP-TFI (1:200, Perseus Proteomics), was diluted in 1\% normal donkey serum and $0.05 \%$ Triton X-100 in PBS and incubated at $4{ }^{\circ} \mathrm{C}$ overnight, and an Alexa Fluor 488-conjugated secondary antibody was used. For Egr1 and Egr2 probes, signals were developed with nitro blue tetrazolium (NBT) and 5-bromo-4-chloro3-indolyl-phosphate (BCIP) substrates (Roche) at room temperature. Images were acquired using an Axio Imager Z1 fluorescence microscope (Carl Zeiss).

Chromatin immunoprecipitation quantitative PCR. Neocortical cells from E14.5 embryonic Foxgl ${ }^{\text {lacZ/+ }}$ heterozygote or Foxgl ${ }^{\text {lacZ } / l a c Z}$ null brains were dissociated into single cells and fixed with $1 \%(\mathrm{v} / \mathrm{v})$ formaldehyde for $8 \mathrm{~min}$ at room temperature, and the reaction was quenched with $125 \mathrm{mM}$ glycine. For chromatin immunoprecipitation (ChIP) assay, chromatin was sheared into an average size of $200 \mathrm{bp}^{63}$. For each reaction, $2 \times 10^{5}$ cells with $2 \mu \mathrm{g}$ antibody were used (rabbit antiBF1 antibody, TaKaRa; rabbit anti-H3K4mel antibody, Abcam; control IgG, Abcam). The isolated DNA was purified using a High Pure PCR Produce Purification Kit (Roche) and quantified by qPCR using SYBR ${ }^{\mathrm{TM}}$ Green PCR Master Mix and an ABI PRISM ${ }^{\odot} 7900$ Sequence Detection System (Applied Biosystems) with E14.5 mouse neocortical genomic DNA as a standard. Primers (Fig. 2f, g) were as follows: PBS1-1F, TGGGAGGGGCAGATAATGGA; PBS1-1R, AGCTTCGGA AACATCGGGTT; PBS1-0F, AGCCGATAATGCATTAGCTCTCA; PBS1-0R, CCGCCGAGTAAAATCGAGGA; PBS1+1 F, GGGGCTGTGCAGGTGTATAT; PBS1+1 R, GGGGCGAGTGAGCAAACATA; PBS2-1F, CCGCACTGAA ACTCTTGTTGG; PBS2-1R, TAGAGCGAGGTCCATGTCCA; PBS2-0F, TTTGTTGGGGGATGGCAGTT; PBS2-0R, ACTTCAACAGTGCCAGCATT; PBS2 + 1 F, TCCAGTGTTTTTGCAGTTGC; PBS2 +1 R, CTGTCCTGTGC GATGCCAC. The specificity of the PCR product of each primer pair was confirmed by the presence of a single band on $2 \%$ agarose gel electrophoresis and a single peak in the dissociation curve. Enrichment (\% Input) was calculated as ChIP DNA/input DNA $\times 100 \%$. Each experiment was repeated at least three times with each replicate producing a similar pattern. The results are shown as the mean \pm SEM of three qPCR triplicates.

Quantitative analysis of callosal axons. To quantify the long-range callosal axons, we first counted the cell number of electroporated cortices using Image 
software (Supplementary Fig. 3a-a'"'). The GFP signal was extracted (3a'), and the background signal was subtracted using a 50-pixel rolling ball radius (Supplementary Fig. 3a"). After the threshold was set to 50-255, Analyze Particles function was performed with size $>5$ and circularity $>0.1$ to automatically count cell number (Supplementary Fig. 3a"'). The intensity of callosal axons in the corpus callosum was measured based on the quantification method using ImageJ software as described previously ${ }^{64}$ (Supplementary Fig. 3b-b) callosum was extracted (Supplementary Fig. 3b), and the background was subtracted using a 5-pixel rolling ball radius (Supplementary Fig. $3 \mathrm{~b}^{\prime}$ ). As callosal axons from similar cortical areas occupied a similar position in the corpus callosum, paired regions in a similar position were selected in each condition (Supplementary Fig. $\left.3 \mathrm{~b}^{\prime \prime}\right)$. The signal was plotted, and the area was measured as GFP intensity (Supplementary Fig. 3b"'). The data were calculated as GFP intensity/the number of cells.

Enhancer reporter assay. For reporter constructs, the $1.2 \mathrm{~kb}$ promoter region of the COUP-TFI gene were cloned into pGL4.1 (Promega) with or without the 550 bp Foxgl binding site PBS1 (Fig. 2h). pRL-TK containing the Renilla luciferase gene was used as an internal standard for transfection efficiency. Reporter plasmid $(0.5 \mu \mathrm{g})$ and pRL-TK $(0.05 \mu \mathrm{g})$ were cotransfected into the U87MG human glioblastoma cell line with Lipofetamine 2000 (Invitrogen) following the Lipofectamine 2000 transfection manual. Two days after transfection, cells were harvested and subjected to the Dual-Luciferase Assay System (Promega). Signals were measured using a 1420ARVOsx-1 luminometer (PerkinElmer Life and Analytical Sciences) according to the manufacturer's standard protocol. Relative luciferase units (RLUs) were calculated as the activity of luciferase/Renilla and normalized to that of the cells transfected with the reporter plasmid containing the COUP-TFI promoter.

Cell isolation and RNA sequencing. The putative somatosensory area was isolated from the embryonic neocortex generated by crossing Neurog 2 CreER/+ heterozygous mice with R26CAG-LSL-tdTomato mice with the indicated $4 \mathrm{OHT}$ administration (Fig. 8b). Cortical neurons were dissociated into single cells using Neuron Dissociation Solution (Wako) following the standard procedure, and then tdTomatopositive cells were purified with a SH800Z Cell Sorter (Sony Biotechnology Inc.). The RNA from isolated cells was extracted using a QIAquick RNeasy Plus Micro Kit (Qiagen). The quantity and quality of RNA were measured by Qubit (Invitrogen) with a Qubit RNA HS Assay Kit and an Agilent 2100 Bioanalyzer with an Agilent Technologies RNA 6000 Pico Kit. Libraries for paired ends were prepared using the Illumina TruSeq Total RNA Sample Prep Kit following the standard procedure. Two biological replicates were analyzed for each condition.

Statistical analysis. The results are shown as the mean \pm SEM with $\geq 3$ biological replicates. For statistical analysis, Student's $t$ test or two-way ANOVA was performed, and significance was recognized as $P$ value $<0.05$. Source data are provided as a Source Data file.

Reporting summary. Further information on research design is available in the Nature Research Reporting Summary linked to this article.

\section{Data availability}

The RNA sequence data that support the findings of this study have been deposited in NCBI Sequence Read Archive with the accession number PRJNA505194. Quantitative data underlying Figs. 2c, g, h, l, 3d, h, m, t, u, 4d, e, o, q, v, 5n-q, 6c, n, p, y, 7b, f, o, 8m, w, x, 9b, l, v and Supplementary Figs. 2e, 4d, 6h, k, s, 7d, o, r, 8b, 9b, c, j are available in the Source Data file. The data that support the findings of this study are available from the corresponding author upon reasonable request.

Received: 5 November 2018 Accepted: 6 June 2019

Published online: 08 August 2019

\section{References}

1. Brodmann, K. Vergleichende Lokalisationslehre der Großhirnrinde (Barth, Leipzig, 1909).

2. Rakic, P. Specification of cerebral cortical areas. Science 241, 170-176 (1988).

3. O'Leary, D. D. Do cortical areas emerge from a protocortex?. Trends Neurosci. 12, 400-406 (1989).

4. Lopez-Bendito, G. \& Molnar, Z. Thalamocortical development: how are we going to get there?. Nat. Rev. Neurosci. 4, 276-289 (2003).

5. Molyneaux, B. J., Arlotta, P., Menezes, J. R. \& Macklis, J. D. Neuronal subtype specification in the cerebral cortex. Nat. Rev. Neurosci. 8, 427-437 (2007).

6. Miyashita-Lin, E. M., Hevner, R., Wassarman, K. M., Martinez, S. \& Rubenstein, J. L. Early neocortical regionalization in the absence of thalamic innervation. Science 285, 906-909 (1999).

7. Sur, M. \& Rubenstein, J. L. Patterning and plasticity of the cerebral cortex. Science 310, 805-810 (2005).
8. Pouchelon, G. et al. Modality-specific thalamocortical inputs instruct the identity of postsynaptic L4 neurons. Nature 511, 471-474 (2014).

9. Wagener, R. J., David, C., Zhao, S., Haas, C. A. \& Staiger, J. F. The somatosensory cortex of reeler mutant mice shows absent layering but intact formation and behavioral activation of columnar somatotopic maps. $J$. Neurosci. 30, 15700-15709 (2010).

10. Vitali, I. et al. Progenitor hyperpolarization regulates the sequential generation of neuronal subtypes in the developing neocortex. Cell 174, 1264-1276 (2018)

11. Oishi, K. et al. Identity of neocortical layer 4 neurons is specified through correct positioning into the cortex. eLife 5, e10907 (2016).

12. Li, H. et al. Laminar and columnar development of barrel cortex relies on thalamocortical neurotransmission. Neuron 79, 970-986 (2013).

13. Vue, T. Y. et al. Thalamic control of neocortical area formation in mice. $J$. Neurosci. 33, 8442-8453 (2013).

14. Srinivasan, K. et al. A network of genetic repression and derepression specifies projection fates in the developing neocortex. Proc. Natl. Acad. Sci. USA 109, 19071-19078 (2012)

15. Kwan, K. Y. et al. SOX5 postmitotically regulates migration, postmigratory differentiation, and projections of subplate and deep-layer neocortical neurons. Proc. Natl. Acad. Sci. USA 105, 16021-16026 (2008).

16. Chen, B. et al. The Fezf2-Ctip2 genetic pathway regulates the fate choice of subcortical projection neurons in the developing cerebral cortex. Proc. Natl. Acad. Sci. USA 105, 11382-11387 (2008).

17. Britanova, O. et al. Satb2 is a postmitotic determinant for upper-layer neuron specification in the neocortex. Neuron 57, 378-392 (2008).

18. McKenna, W. L. et al. Mutual regulation between Satb2 and Fezf2 promotes subcerebral projection neuron identity in the developing cerebral cortex. Proc. Natl. Acad. Sci. USA 112, 11702-11707 (2015).

19. Hevner, R. F. et al. Tbrl regulates differentiation of the preplate and layer 6 . Neuron 29, 353-366 (2001)

20. Kumamoto, T. et al. Foxg1 coordinates the switch from nonradially to radially migrating glutamatergic subtypes in the neocortex through spatiotemporal repression. Cell Rep. 3, 931-945 (2013).

21. Toma, K., Kumamoto, T. \& Hanashima, C. The timing of upper-layer neurogenesis is conferred by sequential derepression and negative feedback from deep-layer neurons. J. Neurosci. 34, 13259-13276 (2014).

22. Molyneaux, B. J., Arlotta, P., Hirata, T., Hibi, M. \& Macklis, J. D. Fezl is required for the birth and specification of corticospinal motor neurons. Neuron 47, 817-831 (2005).

23. Oishi, K., Aramaki, M. \& Nakajima, K. Mutually repressive interaction between Brn1/2 and Rorb contributes to the establishment of neocortical layer 2/3 and layer 4. Proc. Natl. Acad. Sci. USA 113, 3371-3376 (2016).

24. Jabaudon, D., Shnider, S. J., Tischfield, D. J., Galazo, M. J. \& Macklis, J. D. RORbeta induces barrel-like neuronal clusters in the developing neocortex. Cereb. Cortex 22, 996-1006 (2012).

25. Nakagawa, Y. \& O'Leary, D. D. Dynamic patterned expression of orphan nuclear receptor genes RORalpha and RORbeta in developing mouse forebrain. Dev. Neurosci. 25, 234-244 (2003).

26. Belgard, T. G. et al. A transcriptomic atlas of mouse neocortical layers. Neuron 71, 605-616 (2011).

27. Studer, M., Filosa, A. \& Rubenstein, J. L. The nuclear receptor COUP-TFI represses differentiation of Cajal-Retzius cells. Brain Res. Bull. 66, 394-401 (2005).

28. Iwasato, T. et al. Dorsal telencephalon-specific expression of Cre recombinase in PAC transgenic mice. Genesis 38, 130-138 (2004).

29. Zhou, C., Tsai, S. Y. \& Tsai, M. J. COUP-TFI: an intrinsic factor for early regionalization of the neocortex. Genes Dev. 15, 2054-2059 (2001).

30. Armentano, M. et al. COUP-TFI regulates the balance of cortical patterning between frontal/motor and sensory areas. Nat. Neurosci. 10, 1277-1286 (2007).

31. Tomassy, G. S. et al. Area-specific temporal control of corticospinal motor neuron differentiation by COUP-TFI. Proc. Natl. Acad. Sci. USA 107, 3576-3581 (2010)

32. Alfano, C. et al. COUP-TFI promotes radial migration and proper morphology of callosal projection neurons by repressing Rnd2 expression. Development 138, 4685-4697 (2011)

33. Alfano, C., Magrinelli, E., Harb, K., Hevner, R. F. \& Studer, M. Postmitotic control of sensory area specification during neocortical development. Nat. Commun. 5, 5632 (2014).

34. Hanashima, C., Shen, L., Li, S. C. \& Lai, E. Brain factor-1 controls the proliferation and differentiation of neocortical progenitor cells through independent mechanisms. J. Neurosci. 22, 6526-6536 (2002).

35. Miyoshi, G. \& Fishell, G. Dynamic FoxG1 expression coordinates the integration of multipolar pyramidal neuron precursors into the cortical plate. Neuron 74, 1045-1058 (2012)

36. Hatanaka, Y., Namikawa, T., Yamauchi, K. \& Kawaguchi, Y. Cortical divergent projections in mice originate from two sequentially generated, 
distinct populations of excitatory cortical neurons with different initial axonal outgrowth characteristics. Cereb. Cortex 26, 2257-2270 (2016).

37. Klingler, E. et al. A translaminar genetic logic for the circuit identity of intracortically projecting neurons. Curr. Biol. 29, 332-339 (2019).

38. Miyata, T. et al. Asymmetric production of surface-dividing and non-surfacedividing cortical progenitor cells. Development 131, 3133-3145 (2004).

39. Patra, R. C., Blue, M. E., Johnston, M. V., Bressler, J. \& Wilson, M. A. Activitydependent expression of Egr1 mRNA in somatosensory cortex of developing rats. J. Neurosci. Res. 78, 235-244 (2004).

40. Fang, F. et al. The early growth response gene Egr2 (Alias Krox20) is a novel transcriptional target of transforming growth factor-beta that is up-regulated in systemic sclerosis and mediates profibrotic responses. Am. J. Pathol. 178, 2077-2090 (2011).

41. McConnell, S. K. \& Kaznowski, C. E. Cell cycle dependence of laminar determination in developing neocortex. Science 254, 282-285 (1991).

42. Das, G. D. \& Altman, J. Transplanted precursors of nerve cells: their fate in the cerebellums of young rats. Science 173, 637-638 (1971).

43. Guo, C. et al. Fezf2 expression identifies a multipotent progenitor for neocortical projection neurons, astrocytes, and oligodendrocytes. Neuron $\mathbf{8 0}$, 1167-1174 (2013).

44. Bedogni, F. et al. Tbrl regulates regional and laminar identity of postmitotic neurons in developing neocortex. Proc. Natl. Acad. Sci. USA 107, 13129-13134 (2010).

45. Hevner, R. F., Hodge, R. D., Daza, R. A. \& Englund, C. Transcription factors in glutamatergic neurogenesis: conserved programs in neocortex, cerebellum, and adult hippocampus. Neurosci. Res. 55, 223-233 (2006).

46. Britz, O. et al. A role for proneural genes in the maturation of cortical progenitor cells. Cereb. Cortex 16, i138-i151 (2006).

47. Telley, L. et al. Sequential transcriptional waves direct the differentiation of newborn neurons in the mouse neocortex. Science 351, 1443-1446 (2016).

48. Stipursky, J. et al. TGF-betal promotes cerebral cortex radial glia-astrocyte differentiation in vivo. Front. Cell. Neurosci. 8, 393 (2014).

49. Faedo, A. et al. COUP-TFI coordinates cortical patterning, neurogenesis, and laminar fate and modulates MAPK/ERK, AKT, and beta-catenin signaling. Cereb. Cortex 18, 2117-2131 (2008).

50. Brown, K. K. et al. NR2F1 deletion in a patient with a de novo paracentric inversion, $\operatorname{inv}(5)(\mathrm{q} 15 \mathrm{q} 33.2)$, and syndromic deafness. Am. J. Med. Genet. A 149A, 931-938 (2009).

51. Al-Kateb, H. et al. NR2F1 haploinsufficiency is associated with optic atrophy, dysmorphism and global developmental delay. Am. J. Med. Genet. A 161A, 377-381 (2013).

52. Chen, C. A. et al. The expanding clinical phenotype of Bosch-Boonstra-Schaaf optic atrophy syndrome: 20 new cases and possible genotype-phenotype correlations. Genet. Med. 18, 1143-1150 (2016).

53. Boggio, E. M. et al. Visual impairment in FOXG1-mutated individuals and mice. Neuroscience 324, 496-508 (2016).

54. Iwasato, T. et al. Cortex-restricted disruption of NMDAR1 impairs neuronal patterns in the barrel cortex. Nature 406, 726-731 (2000).

55. Hanashima, C., Li, S. C., Shen, L., Lai, E. \& Fishell, G. Foxg1 suppresses early cortical cell fate. Science 303, 56-59 (2004).

56. Zirlinger, M., Lo, L., McMahon, J., McMahon, A. P. \& Anderson, D. J. Transient expression of the bHLH factor neurogenin-2 marks a subpopulation of neural crest cells biased for a sensory but not a neuronal fate. Proc. Natl. Acad. Sci. USA 99, 8084-8089 (2002).

57. Madisen, L. et al. A robust and high-throughput Cre reporting and characterization system for the whole mouse brain. Nat. Neurosci. 13, 133-140 (2010).

58. Cong, L. et al. Multiplex genome engineering using CRISPR/Cas systems. Science 339, 819-823 (2013).

59. Labun, K., Montague, T. G., Gagnon, J. A., Thyme, S. B. \& Valen, E. CHOPCHOP v2: a web tool for the next generation of CRISPR genome engineering. Nucleic Acids Res. 44, W272-W276 (2016).

60. Kawai, S., Takagi, Y., Kaneko, S. \& Kurosawa, T. Effect of three types of mixed anesthetic agents alternate to ketamine in mice. Exp. Anim. 60, 481-487 (2011).
61. Hanashima, C., Fernandes, M., Hebert, J. M. \& Fishell, G. The role of Foxg1 and dorsal midline signaling in the generation of Cajal-Retzius subtypes. J. Neurosci. 27, 11103-11111 (2007)

62. Hou, P. S., Kumamoto, T. \& Hanashima, C. A sensitive and versatile in situ hybridization protocol for gene expression analysis in developing amniote brains. Methods Mol. Biol. 1650, 319-334 (2017).

63. Hou, P. S. et al. LHX2 regulates the neural differentiation of human embryonic stem cells via transcriptional modulation of PAX6 and CER1. Nucleic Acids Res. 41, 7753-7770 (2013).

64. Zhou, J. et al. Axon position within the corpus callosum determines contralateral cortical projection. Proc. Natl. Acad. Sci. USA 110, E2714-E2723 (2013).

\section{Acknowledgements}

The authors thank members of the Hanashima Laboratory for valuable discussions and Q. Wu and D. Campbell for critical reading of the manuscript. The authors thank D. Anderson for the $\mathrm{Neurog}_{2} \mathrm{CreER}$ mice, S. Itohara for the Emx1 ${ }^{\text {cre/t+ }}$ mice, R. Oda for technical assistance, and C. Tanegashima and the technical support staff of the Laboratory for Phyloinformatics at RIKEN BDR for RNA-seq data acquisition. This work was supported by the Grants-in-Aid for Scientific Research on Innovative Areas ("Interplay of Developmental Clock and Extracellular Environment in Brain Formation") JP16H06483 to C.H., JP17H05775 and JP19H04789 to G.M., and Ministry of Education, Culture, Sports, Science and Technology (MEXT) and Japan Society for the Promotion of Science Grants-in-Aid for Scientific Research (KAKENHI) JP16H04798, JP16K14564, JP17F17090 and JP19H03237 to C.H. and JSPS fellowship to P.S.H., and JP17K07102 and JP19H05228 to G.M

\section{Author contributions}

P.S.H. and C.H. initiated the study, designed experiments, analyzed the data, and wrote the paper. P.S.H. conducted experiments and collected the data. G.M. provided materials and comments on manuscript.

\section{Additional information}

Supplementary Information accompanies this paper at https://doi.org/10.1038/s41467019-11043-w.

Competing interests: The authors declare no competing interests.

Reprints and permission information is available online at http://npg.nature.com/ reprintsandpermissions/

Peer review information: Nature Communications thanks Alexander Fleischmann Song-Hai Shi and the other, anonymous, reviewer(s) for their contribution to the peer review of this work. Peer reviewer reports are available.

Publisher's note: Springer Nature remains neutral with regard to jurisdictional claims in published maps and institutional affiliations.

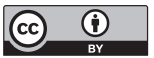

Open Access This article is licensed under a Creative Commons Attribution 4.0 International License, which permits use, sharing, adaptation, distribution and reproduction in any medium or format, as long as you give appropriate credit to the original author(s) and the source, provide a link to the Creative Commons license, and indicate if changes were made. The images or other third party material in this article are included in the article's Creative Commons license, unles indicated otherwise in a credit line to the material. If material is not included in the article's Creative Commons license and your intended use is not permitted by statutory regulation or exceeds the permitted use, you will need to obtain permission directly from the copyright holder. To view a copy of this license, visit http://creativecommons.org/ licenses/by/4.0/

(C) The Author(s) 2019 